Discrete Comput Geom 37:381-407 (2007)

DOI: $10.1007 / \mathrm{s} 00454-006-1280-9$

\title{
Computing Modular Coincidences for Substitution Tilings and Point Sets*
}

\author{
D. Frettlöh ${ }^{1}$ and B. Sing ${ }^{2}$ \\ ${ }^{1}$ Fakultät für Mathematik, Universität Bielefeld, \\ Postfach 100131, 33501 Bielefeld, Germany \\ frettloe@math.uni-bielefeld.de \\ ${ }^{2}$ Department of Mathematics, The Open University, \\ Watton Hall, Milton Keynes, MK7 6AA, England
}

\begin{abstract}
Computing modular coincidences can show whether a given substitution system, which is supported on a point lattice in $\mathbb{R}^{d}$, consists of model sets or not. We prove the computatibility of this problem and determine an upper bound for the number of iterations needed. The main tool is a simple algorithm for computing modular coincidences, which is essentially a generalization of the Dekking coincidence to more than one dimension, and the proof of equivalence of this generalized Dekking coincidence and modular coincidence. As a consequence, we also obtain some conditions for the existence of modular coincidences. In a separate section, and throughout the article, a number of examples are given.
\end{abstract}

\section{Introduction}

Substitution tilings, or substitution point sets, are examples of objects which show high order at both short and long range, even if they are not periodic. This manifests itself in the diffraction spectrum of such a tiling. Like crystals or periodic tilings, some nonperiodic substitution tilings show a diffraction pattern consisting of bright spots- the "Bragg peaks"- only. Such tilings are called pure point diffractive. In general, it is hard to decide whether a given substitution tiling is pure point diffractive. For a special class of tilings the question can be answered for any given example in principle: If the set of difference vectors between tiles spans a discrete point lattice, then there is a condition which is equivalent to pure point diffractivity ([LMS2], see also Theorem 3.3 below). This class covers many well-known examples, such as the chair tiling and the sphinx tiling (being pure point, see, e.g., [So], $[\mathrm{BMS}],[\mathrm{R}]$ and $[\mathrm{LM}]$ ), or the Thue-Morse

\footnotetext{
* B.S. acknowledges financial support by the Cusanuswerk. Both authors express their thanks to the German Research Council, Collaborative Research Center 701.
} 
sequence and the table tiling (being not pure point, see, e.g., [Ma], $[\mathrm{K}]$ and $[\mathrm{R}]$ ). In fact, the condition of [LMS2] may be difficult to check in some cases. Here, we devise a simple algorithm and show the equivalence between the output of this algorithm and the condition mentioned. As a consequence, we obtain results about the computability of the problem and some conditions for the existence (resp. nonexistence) of modular coincidences.

The paper is organized as follows: Section 2 contains notations and basic terms used in this text, the definition of lattice substitution systems and related facts and definitions. Section 3 is dedicated to the main result of [LMS2] (Theorem 3.3), together with the definitions of model sets and modular coincidence. Since we used the notation of [LMS2] wherever it is possible, readers who are familiar with [LMS2] may skip Sections 2 and 3. In Section 4 we give the construction of the coincidence graph, which is a generalization of the Dekking coincidence [D], and establish the equivalence between this coincidence and modular coincidence (Theorem 4.4). From this result, we obtain results about the computability of the problem (Theorems 4.5 and 4.12), as well as some conditions (Corollaries 4.6, 4.7 and 4.9) for the existence (resp. nonexistence) of modular coincidences. Section 5 is a brief remark about the best upper bound for the costs of computing modular coincidences. Section 6 is a collection of examples which illustrate terms and statements of preceding sections and show the usefulness of our results. Finally, three appendices show the connections between this text and the Dekking coincidence, the overlap algorithm in [So] and automatic sequences, respectively.

\section{Basic Definitions, Substitution Systems}

Let us fix some notation. By $\mathbb{N}$ we denote the set of all positive integers. The sum of two sets is to be understood pointwise, i.e., $M+N=\{n+m \mid m \in M, n \in N\}$. The closure of a set $M$ is denoted by $\operatorname{cl}(M)$, the interior of $M$ by $\operatorname{int}(M)$. The cardinality of $A$ is denoted by \#A (where \# $(\varnothing)=0$ ). By $\mathbb{B}^{d}$ we denote the closed unit ball $\{x \mid\|x\| \leq 1\}$ in $\mathbb{R}^{d}$. A tile is a nonempty compact set $T \subset \mathbb{R}^{d}$ with the property that $\operatorname{cl}(\operatorname{int}(T))=T$. A tiling of $\mathbb{R}^{d}$ is a collection of tiles $\left\{T_{n}\right\}_{n \in \mathbb{N}}$ which covers $\mathbb{R}^{d}$ and contains no overlapping tiles, i.e., $\operatorname{int}\left(T_{k}\right) \cap \operatorname{int}\left(T_{n}\right)=\varnothing$ for $k \neq n$. A tiling $\mathcal{T}$ is called nonperiodic if the only solution of $\mathcal{T}+x=\mathcal{T}$ is $x=0$. If the tiles of a tiling belong to finitely many translation classes $\left[T_{1}\right],\left[T_{2}\right], \ldots,\left[T_{m}\right]$, then the class representatives $T_{1}, T_{2}, \ldots, T_{m}$ are called prototiles. If necessary, one may consider two congruent prototiles as different by labeling them with their type. Given a set of prototiles $T_{i}$, one can describe tilings-or parts of tilings-by a set

$$
\left\{T_{1}+v_{1}^{(1)}, T_{1}+v_{2}^{(1)}, \ldots, T_{2}+v_{1}^{(2)}, T_{2}+v_{2}^{(2)}, \ldots, T_{m}+v_{1}^{(m)}, \ldots\right\} \quad\left(v_{i}^{(j)} \in \mathbb{R}^{d}\right),
$$

or shortly by $\left\{T_{1}+V_{1}, T_{2}+V_{2}, \ldots, T_{m}+V_{m}\right\}$-where $V_{i}$ contains all $v_{k}^{(i)}$ of (1)—or even shorter by a position vector $\mathbf{V}=\left(V_{1}, V_{2}, \ldots, V_{m}\right)^{T}$.

Nonperiodic tilings of long range order can easily be generated by a substitution. The idea is, basically, to give a set of prototiles and a rule on how to enlarge them, and then to dissect them into tiles congruent to the original prototiles (see Example 7 in Section 6). Formally, this can be done by a matrix function system, resp. by a substitution system. 
Definition 2.1. A matrix function system $(M F S)$ is an $m \times m$ matrix $\Phi=\left(\Phi_{i j}\right)_{1 \leq i, j \leq m}$, where each $\Phi_{i j}$ is a finite (possibly empty) set of mappings $x \mapsto Q x+a_{i j k}$, where $Q$ is an expansive linear map, $1 \leq i, j \leq m, 1 \leq k \leq \#\left(\Phi_{i j}\right), a_{i j k} \in \mathbb{R}^{d}$. An MFS $\Phi$ is called primitive if its substitution matrix $S(\Phi)=\left(\#\left(\Phi_{i j}\right)\right)_{1 \leq i, j \leq m}$ (an $m \times m$ matrix with non-negative integer entries) is primitive, i.e., $S^{k}$ has only strictly positive entries for some $k \in \mathbb{N}$.

Note that we can define $\Phi^{k}$ (for $k \in \mathbb{N}$ ) inductively by the composition of maps, i.e., $\Phi^{k+1}=\Phi^{k} \circ \Phi=\left(\Phi^{k} \circ \Phi\right)_{1 \leq i, j \leq m}=\left(\bigcup_{\ell=1}^{m}\left(\Phi^{k}\right)_{i \ell} \circ(\Phi)_{\ell j}\right)_{1 \leq i, j \leq m}$ where

$$
\left(\Phi^{k}\right)_{i \ell} \circ \Phi_{\ell j}= \begin{cases}\varnothing & \text { if }\left(\Phi^{k}\right)_{i \ell}=\varnothing \text { or } \Phi_{\ell j}=\varnothing, \\ \left\{g \circ f \mid g \in\left(\Phi^{k}\right)_{i \ell}, f \in \Phi_{\ell j}\right\} & \text { otherwise. }\end{cases}
$$

Clearly, we have $S\left(\Phi^{k}\right) \leq S(\Phi)^{k}$. Note also that the linear part of the affine mappings in $\Phi^{k}$ is given by $x \mapsto Q^{k} x$. Now we can apply the MFS $\Phi$ to a position vector $\mathbf{V}$. That is, if $\mathbf{V}=\left(V_{1}, \ldots, V_{m}\right)$ is a position vector as above, then $\Phi \mathbf{V}=\left(W_{1}, \ldots, W_{m}\right)$, $W_{i}=\bigcup_{j=1}^{m} \Phi_{i j}\left(V_{j}\right)=\bigcup_{j=1}^{m} \bigcup_{f \in \Phi_{i j}} f\left(V_{j}\right)$.

Definition 2.2. A substitution system is a pair $(\mathbf{V}, \Phi)$, where $\mathbf{V}=\left(V_{1}, V_{2}, \ldots, V_{m}\right)^{T}$ is a fixed point of the matrix function system $\Phi$ (i.e., $\Phi \mathbf{V}=\mathbf{V}$ ), such that all $V_{i}$ are mutually disjoint and the unions in

$$
V_{i}=\bigcup_{j=1}^{m} \Phi_{i j}\left(V_{j}\right)=\bigcup_{j=1}^{m} \bigcup_{f \in \Phi_{i j}} f\left(V_{j}\right)
$$

are pairwise disjoint for all $1 \leq i \leq m$. If $\operatorname{supp}(\mathbf{V})=\bigcup_{i=1}^{m} V_{i}$ is a lattice, then $(\mathbf{V}, \Phi)$ is called a lattice substitution system (LSS) with $m$ components.

Now the relation between substitution systems and substitution tilings becomes clear: A set of tiles is represented by a vector $\mathbf{V}$ whose entries $V_{i}$ are point sets (where each point encodes the position of one tile). The substitution applied to this set of tiles yields a larger set of tiles, which is represented by the vector $\Phi \mathbf{V}$.

By the action of a tile substitution, each tile $T$ is enlarged by $Q$, then dissected into some tiles of type $\left[T_{1}\right], \ldots,\left[T_{m}\right]$. If $T \in\left[T_{i}\right]$, then $Q T$ is dissected into \# $\left(\Phi_{1 i}\right)$ tiles congruent to $T_{1}, \#\left(\Phi_{2 i}\right)$ tiles congruent to $T_{2}$ and so on. The translational part of the affine maps in $\Phi$ determines the exact location of these tiles inside the enlarged tile. In this way, $(\mathcal{T}, \Phi)$ can be considered a substitution system. Wherever we emphasize the fact that $(\mathcal{T}, \Phi)$ describes a tiling, we call $\mathcal{T}$ a substitution tiling and $\Phi$ a tile substitution. For examples of the relation between tile substitutions and substitutions on point sets, we refer to Example 7 of Section 6. The next lemma reads essentially: The translate of an LSS $(\mathbf{V}, \Phi)$ by a $t \in \operatorname{supp}(\mathbf{V})$ is again an LSS.

Lemma 2.3. Let $(\mathbf{V}, \Phi)$ be an LSS, let $t \in \operatorname{supp}(\mathbf{V})$ and let $(\mathbf{V}-t, \Phi+Q t-t)$ be defined by $\mathbf{V}-t=\left(V_{1}-t, \ldots, V_{m}-t\right)$ and $\Phi+Q t-t=\left(\Phi_{i j}+Q t-t\right)_{i j}, \Phi_{i j}+Q t-t=$ $\left\{\varphi+Q t-t \mid \varphi \in \Phi_{i j}\right\}$. Then $(\mathbf{V}-t, \Phi+Q t-t)$ is also an LSS. 
Proof. A simple calculation (see Definition 2.2) yields $(\Phi+Q t-t)(\mathbf{V}-t)=\mathbf{V}-t$ if and only if $\Phi(\mathbf{V})=\mathbf{V}$. Since $t \in \operatorname{supp}(\mathbf{V})$, it follows that $\operatorname{supp}(\mathbf{V}-t)$ is a lattice, thus all conditions for lattice substitution systems are satisfied.

If $(\mathbf{V}, \Phi)$ is an LSS, then the set $\mathbf{V}$ in Definition 2.2 consists of point sets $V_{i}$. Rather than considering $\mathbf{V}$ as a subset of $\mathbb{R}^{d m}$, we may think of $\mathbf{V}$ as a point set in $\mathbb{R}^{d}$, where each point has got one color (out of $m$ colors altogether). Such a single point of $\mathbf{V}$ with color $j$, located at $x \in \mathbb{R}^{d}$, is of the form

$$
\{(\varnothing, \ldots, \varnothing, \underbrace{\{x\}}_{j \text { th entry }}, \varnothing, \ldots, \varnothing)\},
$$

which is a somewhat inconvenient notation. Therefore, we avoid considering such single points as much as possible. If such a single colored point located at $x$ of type $j$ is considered, we use the notation "V $\cap\{x\}, x$ is of type $j$ " (or of color $j$ ).

In this paper we consider only the primitive LSS $(\mathbf{V}, \Phi)$. This implies that each $V_{i}$ is a Delone set, and we call $\mathbf{V}$ a multi-component Delone set (see Section 2 of [LMS1], where the name Delone multiset is used; however, the name "multiset" in this context is misleading).

Definition 2.4. A set $V \subset \mathbb{R}^{d}$ is a Delone set (or Delaunay set) if it satisfies the following two conditions:

(1) $V$ is uniformly discrete: There is a value $r$ such that each open ball of radius $r$ contains at most one point of $V$.

(2) $V$ is relatively dense: There is a value $R$ such that each closed ball of radius $R$ contains at least one point of $V$.

Note that the maximal such radius $r$ is the packing radius for $V$, while the minimal value of $R$ is the covering radius of $V$.

We should mention that every primitive substitution tiling gives rise to a primitive substitution system in a quite natural way, see (1) and comments there. In the other direction, this is less obvious. In the case of LSS, one simple way is the following: Assign to each point $x$ of type $i$ in a multi-component Delone set a tile $x+F$ of type $i$, where $F$ is a fundamental domain of the lattice. Then it is trivial to read off the substitution for the tiling from the MFS for the multi-component Delone set. Note that we do not require the tile substitution to be self-similar in the sense of [So]. For details about the subtleties of the relation between point substitutions and tile substitutions, see Section 3.2 of [LMS2]. The property which describes this relation is given in the following definition. It works both for tilings and for multi-component Delone sets. In order to express it, we denote by $C_{r}(x, A)$ the finite portion of the structure $A$ in a ball of radius $r$ around $x$, the $r$-cluster at $x$. If $A$ is a tiling, then $C_{r}(x, A):=\left\{T \in A \mid T \cap\left(r \mathbb{B}^{d}+x\right) \neq \varnothing\right\}$. If $A=\left(A_{1}, \ldots, A_{m}\right)$ is a multi-component Delone set, then $C_{r}(x, A):=\left(A_{1} \cap\left(r \mathbb{B}^{d}+x\right), \ldots, A_{m} \cap\left(r \mathbb{B}^{d}+x\right)\right)$.

Definition 2.5. Let $A, B$ be two multi-component Delone sets, or two tilings, or one multi-component Delone set and one tiling. $B$ is locally derivable (with radius $r$ ) from 
$A$, if, for all $x, y$ in $\mathbb{R}^{d}$, one has

$$
C_{r}(x, A)=C_{r}(y, A)+(x-y) \quad \Longrightarrow \quad C_{0}(x, B)=C_{0}(y, B)+(x-y) .
$$

If $B$ is locally derivable from $A$ and $A$ is locally derivable from $B$, then $A$ and $B$ are mutually locally derivable (MLD), see [BSJ].

For examples of a tiling and its corresponding substitution system being MLD see Example 7 in Section 6. Another property which is frequently used is the following.

Definition 2.6. A tiling $A$, or a multi-component Delone multi-set $A$, is called repetitive, if for every $r>0$ there is $R>0$ such that each $R$-cluster in $A$ contains a translate of each $r$-cluster in $A$.

\section{Model Sets}

The objects we consider in this paper are primitive lattice substitution systems $(\mathbf{V}, \Phi)$ and tilings which are MLD to a primitive LSS. To exclude certain pathological cases, we assume further that all $r$-clusters in $\mathbf{V}$ are contained in some $\Phi^{k}(\mathbf{v})$, where $k \in \mathbb{N}$ and $\mathbf{v}$ is a single point of some type $i$. In particular, this ensures that $\mathbf{V}$ is repetitive. Moreover, the dynamical system associated to $\mathbf{V}$ is always minimal, see the remark at the end of this section or [So] for details.

A central question is whether such a substitution system consists of model sets or not; and whether the diffraction spectrum of such a substitution pattern is pure point or not. If $\mathbf{V}$ is fully periodic (i.e., there are $d$ linearly independent translation vectors such that $\mathbf{V}$ is invariant under these translations), this is trivial. The question becomes interesting if $\mathbf{V}$ is not fully periodic. A theorem by Schlottmann [Sc, Theorem 4.5] states that all regular model sets are pure point diffractive. We first recall what a model set is, see [Mo].

Definition 3.1. A cut and project scheme consists of a collection of spaces and mappings as follows:

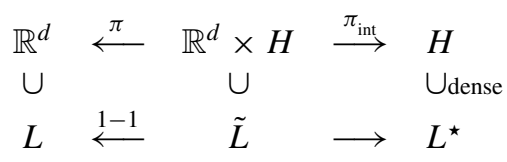

where $H$ is a locally compact Abelian group, $\tilde{L} \subset \mathbb{R}^{d} \times H$ is a lattice, i.e., a discrete subgroup for which the quotient group $\left(\mathbb{R}^{d} \times H\right) / \tilde{L}$ is compact, and $\pi, \pi_{\text {int }}$ denote the canonical projections, where $\pi_{\text {int }}(\tilde{L})=L^{\star}$ is dense in $H$ and the restriction $\left.\pi\right|_{\tilde{L}}$ is injective (and therefore bijective as a map from $\tilde{L}$ to $L=\pi(\tilde{L})$ ). We call $\mathbb{R}^{d}$ the direct space (or physical space) and $H$ the internal space.

A model set (or cut and project set) in $\mathbb{R}^{d}$ is a subset of $\mathbb{R}^{d}$ which (up to translation) is of the form $\Lambda(\Omega)=\left\{\pi(x) \mid x \in \tilde{L}, \pi_{\text {int }}(x) \in \Omega\right\}$ for some cut and project scheme, where $\Omega \subset H$ has non-empty interior and compact closure. We call $\Omega$ the window of the model set.

A model set $\Lambda(\Omega)$ is regular if the boundary $\partial \Omega=\operatorname{cl}(\Omega) \backslash \operatorname{int}(\Omega)$ is of Haar measure 0 . 
Note that one can define the so-called star map $x^{\star}$ of $x \in L$ by $x^{\star}=\pi_{\text {int }}\left(\left.\pi\right|_{\tilde{L}} ^{-1}(x)\right)$, justifying the notation $L^{\star}$ above.

To an LSS, one can naturally associate a cut and project scheme (see Definition 3.2 below for the definition of $\left.L^{\prime}\right)$. The internal space is then given by $H=\left(L / L^{\prime}\right) \times$ $\lim _{\leftarrow k} L^{\prime} / Q^{k} L^{\prime}$, where $\lim _{\leftarrow k} L^{\prime} / Q^{k} L^{\prime}$ denotes the $Q$-adic completion of $L^{\prime}$ (a profinite group), see Section 5.1 of [LMS2], and Examples 1 and 2 in Section 6. Furthermore, the lattice $\tilde{L}$ also arises naturally as diagonal embedding of $L$ in $\mathbb{R}^{d} \times H$, i.e., $\tilde{L}=\{(t, t) \mid$ $t \in L\}$. So only the existence and shape of a window is in question. For this, a central role is played by the notion of a modular coincidence.

Definition 3.2 (see [LM] and [LMS2]). Let $(\mathbf{V}, \Phi)$ be an LSS and let $L=\operatorname{supp}(\mathbf{V})$. Let $L^{\prime}:=L_{1}+L_{2}+\cdots+L_{m}$, where $L_{i}:=\left\langle V_{i}-V_{i}\right\rangle_{\mathbb{Z}}$. For $a \in L$, let

$$
\begin{aligned}
\Phi_{i j}[a] & :=\left\{\varphi \in \Phi_{i j} \mid \varphi(y) \equiv a \bmod Q L^{\prime}, \text { where } V_{j} \subset y+L^{\prime}\right\} \\
& =\left\{\varphi \in \Phi_{i j} \mid \varphi\left(V_{j}\right) \subset a+Q L^{\prime}\right\} .
\end{aligned}
$$

Furthermore, let

$$
\Phi[a]:=\bigcup_{1 \leq i, j \leq m} \Phi_{i j}[a] .
$$

$(\mathbf{V}, \Phi)$ admits a modular coincidence relative to $Q L^{\prime}$, if $\Phi[a]$ is contained entirely in one row of $\Phi$ for some $a \in L$, i.e., if $\left(a+Q L^{\prime}\right) \subset V_{i}$ for some $1 \leq i \leq m$.

For an illustration of this concept, see Example 4 in Section 6 with an explicit calculation of a modular coincidence in a particular case. (At this point, consider just the first part of the example, before the first occurrence of $\Psi$.)

Remark. Since $Q(L) \subseteq L$, one also has $Q\left(L^{\prime}\right) \subseteq L^{\prime}$ (see Section 5.1 of [LMS2]). Furthermore, $L / L^{\prime}$ is finite, i.e., $L^{\prime}$ partitions $L$ in finitely many cosets. By the definition of $L^{\prime}$, each $V_{j}$ is a subset of one such coset (it is possible that more than one $V_{j}$ are subsets of a fixed coset). Clearly, we have $V_{j} \subset y+L^{\prime}$ for $y \in V_{j}$. Since $\varphi \in \Phi_{i j}$ is affine, we get $\varphi\left(V_{j}\right) \subset \varphi\left(y+L^{\prime}\right)=\varphi(y)+Q L^{\prime}$. Therefore, $\Phi[a]$ partitions $\Phi$ into congruence classes induced by the cosets $L / Q L^{\prime}$. Note that $\Phi[a] \neq \varnothing$ for all $a \in L$. Note also that even in the one-dimensional case, not all $L_{i}$ have to be equal, see Example 1 in Section 6.

The answer to the questions about an LSS being a model set and being pure point diffractive is now given in the following theorem.

Theorem 3.3 [LMS2, Theorem 5.12]. Let $(\mathbf{V}, \Phi)\left(\right.$ with $\left.\mathbf{V}=\left(V_{1}, \ldots, V_{m}\right)\right)$ be a primitive LSS, $L^{\prime}=L_{1}+\cdots+L_{m}$, where $L_{i}:=\left\langle V_{i}-V_{i}\right\rangle_{\mathbb{Z}}$. Then the following claims are equivalent:

(1) Each $V_{i}$ is a regular model set.

(2) There is a $k \in \mathbb{N}$ such that a modular coincidence relative to $Q^{k} L^{\prime}$ occurs in $\Phi^{k}$.

(3) $\mathbf{V}$ is pure point diffractive (meaning that each $V_{i}$ is pure point diffractive).

Essentially, this theorem tells us that it is sufficient to compute powers of $\Phi$, until there is a map $\varphi$ which occurs in one row of $\Phi^{k}$ only. Alternatively, it follows from 
[LMS2] that it is enough to find one sublattice $a+Q^{k} L^{\prime}$ which lies entirely in a single $V_{i}$, to ensure that each $V_{j}$ is a regular model set. At this point, it is difficult to estimate an upper bound for $k$, or even to decide whether the derived algorithms will terminate, assuming that $\mathbf{V}$ is not a model set. An answer is given by Theorem 4.5 below.

Note that the above construction does not yield the "minimal" possible internal space, see Example 2 in Section 6.

Remark. To a multi-component Delone set $\mathbf{V}$ (or a tiling $\mathcal{T}$ ), as obtained via a primitive substitution, one can associate a compact metric space $\mathbb{X}_{\mathbf{V}}\left(\mathbb{X}_{\mathcal{T}}\right)$. Here, $\mathbb{X}_{\mathbf{V}}:=\operatorname{cl}(\{\mathbf{V}-$ $\left.t \mid t \in \mathbb{R}^{d}\right\}$ ), i.e., it is the closure of the set of all translates of $\mathbf{V}$ in the space of all multi-component Delone sets, where the metric yields the following definition of closeness: Two multi-component Delone sets $\mathbf{V}_{1}, \mathbf{V}_{2}$ are close to each other if they coincide, after a small shift, inside a large ball around the origin. This compact space together with the translation action $\mathbb{R}^{d}$ yields a topological dynamical system $\left(\mathbb{X}_{\mathbf{V}}, \mathbb{R}^{d}\right)$. If the eigenfunctions of this action span a dense subspace of $L^{2}\left(\mathbb{X}_{\mathbf{V}}\right)$, then we say that $\mathbf{V}$ has a pure point dynamical spectrum. In the cases considered here, $\mathbf{V}$ (resp. $\mathcal{T}$ ) has a pure point dynamical dynamical spectrum iff it is pure point diffractive. This equivalence is often used in the literature. In fact, this equivalence plays an essential role in the proof of Theorem 3.3. For details on dynamical systems and this last connection see [LMS1], [LMS2], [G] and [BL].

\section{Main Results}

In connection with modular coincidence, we define the following subsets of $\{1, \ldots, m\}$ :

$$
\Psi_{k}[a]:=\left\{i \mid\left(a+Q^{k} L^{\prime}\right) \cap V_{i} \neq \varnothing\right\} \quad\left(a \in L / Q^{k} L^{\prime}\right) .
$$

The following properties are obvious:

1. $\Psi_{k}[a] \subset\{1, \ldots, m\}$ and $1 \leq \#\left(\Psi_{k}[a]\right) \leq m$.

2. $a+Q^{k} L^{\prime} \subset \bigcup_{i \in \Psi_{k}[a]} V_{i}$.

3. ( $\mathbf{V}, \Phi)$ admits a modular coincidence relative to $Q^{k} L^{\prime}$ iff there is an $a \in L / Q^{k} L^{\prime}$ such that $\#\left(\Psi_{k}[a]\right)=1$.

4. For $\bar{z} \in L / L^{\prime}$, denote by $z$ an element of the associated coset. Then $\Psi_{0}[z]$ is a partition of $\{1, \ldots, m\}$ as $z$ runs through $L / L^{\prime}$.

5. By $Q^{k+1} L^{\prime} \subset Q^{k} L^{\prime}$ we have $\Psi_{k}[a]=\bigcup_{z \equiv a \bmod Q^{k} L^{\prime}} \Psi_{k+1}[z]$.

Already these properties can sometimes be used directly to decide on the (non-) existence of modular coincidences, see Example 3 in Section 6.

Note that the sets $\Psi_{k}[a]$ can be calculated iteratively:

$$
\begin{aligned}
& \Psi_{k+1}[b]=\left\{i \mid\left(b+Q^{k+1} L^{\prime}\right) \cap V_{i} \neq \varnothing\right\} \\
& \stackrel{\text { Def. } 2.2}{=}\left\{i \mid\left(b+Q^{k+1} L^{\prime}\right) \cap\left(\bigcup_{j} \bigcup_{\varphi \in \Phi_{i j}[b]} \varphi\left(V_{j}\right)\right) \neq \varnothing\right\}
\end{aligned}
$$




$$
\begin{aligned}
& =\left\{i \mid \bigcup_{j} \bigcup_{\left.\varphi \in \Phi_{i j} b b\right]}\left(\left(b+Q^{k+1} L^{\prime}\right) \cap \varphi\left(V_{j}\right)\right) \neq \varnothing\right\} \\
& =\left\{i \mid \bigcup_{j} \bigcup_{\left.\varphi \in \Phi_{i j} b b\right]} \varphi\left(\left(a+Q^{k} L^{\prime}\right) \cap V_{j}\right) \neq \varnothing,\right. \\
& \left.\quad \text { where } \varphi(a) \equiv b \bmod Q^{k+1} L^{\prime}\right\} \\
& =\quad\left\{i \mid \text { there exist } j, \varphi \in \Phi_{i j}[b] \text { and } a \in L \text { s.t. } \varphi(a) \equiv b \bmod Q^{k+1} L^{\prime}\right. \\
& \text { and } \left.j \in \Psi_{k}[a]\right\},
\end{aligned}
$$

where the fourth equation is due to the following: If $\left(b+Q^{k+1} L^{\prime}\right) \cap \varphi\left(V_{j}\right) \neq \varnothing$, then there exists an $a$ such that $\varphi(a) \equiv b \bmod Q^{k+1} L^{\prime}$.

For a fixed $\Psi_{k}[a]$, we call each $\Psi_{k+1}[b]$ which is determined by the previous equation a child of $\Psi_{k}[a]$. Conversely, for a fixed $\Psi_{k+1}[b]$, we call all $\Psi_{k}[a]$ which appear in the previous equation the parents of $\Psi_{k+1}[b]$.

Example 4 in Section 6 illustrates this concept. This example also inspires the following notation: We fix a system of representatives (which includes 0 ) in $L$ of $L / Q L$, denoted by $\alpha_{i}$. Then we can code every $a \in L$ by a finite sequence $a=\left(\alpha_{1}, \alpha_{2}, \ldots, \alpha_{n}\right)$ (the $Q$ adic expansion of $a$ ), where $a \in \alpha_{1}+Q L, a-\alpha_{1} \in \alpha_{2}+Q^{2} L, a-\alpha_{1}-Q \alpha_{2} \in \alpha_{3}+Q^{3} L$, etc.

Definition 4.1. A primitive LSS $(\mathbf{V}, \Phi)$ is called admissible if all mappings in $\Phi$ have translational parts of the form $\left(\alpha_{i j k}, 0,0, \ldots\right)$, where $\alpha_{i j k}$ is an element of a fixed system of representatives for $L / Q L$.

Note that, by the definition of an LSS, all such translational parts appear exactly once in each column of $\Phi$ in the admissible case. The admissible primitive LSSs are those to be considered in what follows. Later we discuss nonadmissible substitution systems (Theorem 4.12). For an example of a nonadmissible primitive LSS, see Example 5 in Section 6.

In the following, we introduce a simple method for computing modular coincidences in the admissible case.

Definition 4.2. The coincidence graph $G_{(\mathbf{V}, \Phi)}$ of a primitive LSS $(\mathbf{V}, \Phi)$ is a directed (multi-)graph, where the vertices represent sets $\Psi_{k}[a]$. If $\Psi_{k}[a]$ and $\Psi_{\ell}[b]$ are equal as sets, then both are represented by the same vertex. We mark the $\left[L: L^{\prime}\right]$ vertices $\Psi_{0}[a]$ as "base points" (e.g., these vertices are represented by a double circle instead of an ordinary circle). We draw an edge from $v_{1}$ to $v_{2}$ iff $v_{2}$ is a child of $v_{1}$. Therefore, there are at most $|\operatorname{det} Q|$ outgoing edges at each vertex.

Of course, one can also label the edges by the element $z \in L^{\prime} / Q L^{\prime}$ which induces the parent/child relation. This is useful if one wants to analyze the substitution further, 
e.g., determine the windows of a model set explicitly. In this case, multiple edges are possible, and there are exactly $|\operatorname{det} Q|$ outgoing edges at each vertex. For examples of coincidence graphs, see Examples 7 and 9 in Section 6.

The practical construction of a coincidence graph works as follows. Let us introduce the following notation: For an (admissible) LSS $(\mathbf{V}, \Phi)$ and fixed $j$, denote by $\Phi(j)_{z}$ the letter $i$ for which $Q x+z \in \Phi_{i j}$ (where $z \in L / Q L^{\prime}$ ). By definition, this is (for every $j$ and $z$ ) exactly one letter. Then we obtain the coincidence graph as follows: We start with the base points $\Psi_{0}[a]\left(a \in L / L^{\prime}\right)$. Suppose $\left\{s_{1}, \ldots, s_{\ell}\right\}=\Psi_{0}[a]$ for some $a$, then there is a directed edge to the vertex representing $\left\{\Phi\left(s_{1}\right)_{z}, \ldots, \Phi\left(s_{\ell}\right)_{z}\right\}$ for each $z$. From these new vertices, we proceed further (to the vertices $\left.\left\{\Phi^{2}\left(s_{1}\right)_{z}, \ldots, \Phi^{2}\left(s_{\ell}\right)_{z}\right\}\right)$ until no further edges and vertices have to be added. This graph is then the coincidence graph.

Lemma 4.3. Let $(\mathbf{V}, \Phi)$ be an admissible primitive substitution system. If $\Psi_{k}[a]=$ $\Psi_{\ell}[c]$, then $\Psi_{k}[a]$ and $\Psi_{\ell}[c]$ have the same set of children.

Proof.

Case $L=L^{\prime}$. Here $a$ in $\Psi_{k}[a]$ is actually a representative of $L / Q^{k} L$, wherefore we can write $\left(\alpha_{a_{1}}, \ldots, \alpha_{a_{k}}\right)$ for $a$. Similarly, we can write $\left(\alpha_{c_{1}}, \ldots, \alpha_{c_{\ell}}\right)$ for $c$. An (admissible) map $\varphi(x)=Q x+\left(\alpha_{i j k}, 0,0, \ldots\right)$ yields (with these identifications) $\varphi(a)=$ $\left(\alpha_{i j k}, \alpha_{a_{1}}, \ldots, \alpha_{a_{k}}\right)$ and $\varphi(c)=\left(\alpha_{i j k}, \alpha_{c_{1}}, \ldots, \alpha_{c_{\ell}}\right)$. Now, since $\Psi_{k}[a]=\Psi_{\ell}[c]$, the same maps operate on $a+Q^{k} L$ as well as on $c+Q^{\ell} L$, and we get $\Psi_{k+1}[t+Q a]=\Psi_{\ell+1}[t+Q c]$ (where $t$ is an associated translational part). Furthermore, we observe in the admissible case that each child has exactly one parent $\left(\Psi_{k+1}\left[\left(\alpha_{1}, \ldots, \alpha_{k+1}\right)\right]\right.$ has parent $\left.\Psi_{k}\left[\left(\alpha_{2}, \ldots, \alpha_{k+1}\right)\right]\right)$. This proves the lemma for $L=L^{\prime}$.

Case $L \neq L^{\prime}$. Since each set $\Psi^{k}[a]$ determines the representative of $L / L^{\prime}$ uniquely, we can simply index the position by the $Q$-adic expansion relative to $L^{\prime}$. This reduces this case to the previous one.

It follows that we need to compute children only as long as we find sets that did not occur before. This justifies the representation of equal children $\Psi_{k}[a]=\Psi_{\ell}[b]$ by a single vertex in Definition 4.2. As a consequence, we obtain the following result.

Theorem 4.4. Let $(\mathbf{V}, \Phi)$ be an admissible primitive LSS. Then $(\mathbf{V}, \Phi)$ admits a modular coincidence iff at least one vertex of the coincidence graph $G_{(\mathbf{V}, \Phi)}$ represents a singleton set. The length of the shortest path from one of the vertices $\Psi_{0}[a]$ to this singleton set is the minimal $k$ such that a modular coincidence relative to $Q^{k} L^{\prime}$ occurs in $\Phi^{k}$.

The claim follows immediately from Lemma 4.3 and the construction of $\Psi$. This result yields an upper bound for the number of iterations needed to compute modular coincidences.

Theorem 4.5. Let $(\mathbf{V}, \Phi)$ be an admissible primitive LSS with $m \geq 2$ components. If $\Phi^{k}$ admits no modular coincidence for $k \leq 2^{m}-m-2$, then $\Phi^{k}$ admits no modular coincidence for any $k \in \mathbb{N}$. 
Proof. As described above, it is possible to determine the sets $\Psi_{k}[a]$ iteratively. The $\operatorname{LSS}\left(\mathbf{V}, \Phi^{k}\right)$ admits a modular coincidence iff there is an $a$ such that $\Psi_{k}[a]$ is a singleton set.

From Lemma 4.3, it follows that we need to compute children until no new sets appear. Since every child is a nonempty subset of $\{1,2, \ldots, m\}$, after at most $2^{m}-2$ iterations all possible sets have occurred. (We count the determination of $\Psi_{0}[\cdot]$ as the zeroth iteration step. So $\Psi_{1}[\cdot]$ corresponds to $\Phi^{1}$.) Moreover, after at most $2^{m}-m-2$ iterations either a set with just one element occurred, or, by Lemma 4.3, it will never occur. By Theorem 4.4, this is equivalent to the claim of the theorem.

As a consequence of Theorem 4.4, we obtain some conditions for $(\mathbf{V}, \Phi)$ admitting a modular coincidence. By Theorem 3.3, this translates to necessary conditions for $\mathbf{V}$ consisting of model sets, resp. being pure point diffractive.

Corollary 4.6. Let $(\mathbf{V}, \Phi)$ be an admissible primitive LSS. If there is an a such that $\Psi_{0}[a]$ is a singleton set, then $\mathbf{V}$ consists of model sets.

Proof. This is an immediate consequence of Theorem 4.4: $\Psi_{0}[a]$ is already a singleton set, thus, by the construction of $\Psi$, we obtain $V_{i}=a+L^{\prime}$ for some $1 \leq i \leq m$. By the remark following Theorem 3.3, $\mathbf{V}$ consists of model sets.

Corollary 4.7. Let $(\mathbf{V}, \Phi)$ be an admissible primitive LSS, such that no $\Psi_{0}[a]$ is a singleton set. If $\mathbf{V}$ consists of model sets, then $\Phi$ admits at least one pairwise coincidence in some $\Psi_{0}[a]$, i.e., there are $j \neq k$ such that $j, k \in \Psi_{0}[a]$ for some $a$ and $\Phi_{i j} \cap \Phi_{i k} \neq \varnothing$ for some $i$.

Proof. Assume that $\Phi$ admits no pairwise coincidence in any $\Psi_{0}[a]$. Therefore, and because any set $\Psi_{0}[a]$ contains more than one element, any child $\Psi_{1}[Q a+z]$ contains more than one element. Inductively, no set $\Psi_{k}[\cdot]$ is a singleton set.

Surprisingly, the last corollary applies to a large number of substitutions found in the literature, showing that they do not consist of model sets, since they admit no pairwise coincidence (see Examples 3, 7 and 8 in Section 6). In Definition 1.2 of [Fra2], the author defines bijective substitutions. This definition reads in our notation as follows.

Definition 4.8. Let $(\mathbf{V}, \Phi)$ be an admissible primitive LSS. If each $\varphi$ occurring in $\Phi$ appears exactly once in each row, then $(\mathbf{V}, \Phi)$ is called bijective substitution (system).

The following statement is mentioned in Section 3.3 of [Fra2], but without proof. Here we obtain the result quite easily.

Corollary 4.9. Let $(\mathbf{V}, \Phi)$ be an admissible primitive LSS, such that $\Phi$ is bijective and $\mathbf{V}$ is nonperiodic. Then $\mathbf{V}$ does not consist of model sets, therefore $\mathbf{V}$ is not pure point diffractive. 
Proof. The proof is an application of Corollary 4.7. $\Phi$ is bijective, thus $\Psi$ admits no pairwise coincidence at all. All we need to show is that no $\Psi_{0}[a]$ is a singleton set.

First we note that in $\mathbf{V}$ all types of points occur with the same frequency, if $\mathbf{V}$ arose from a bijective substitution: Since $(\mathbf{V}, \Phi)$ is bijective, every $\varphi$ appears exactly once in each row of $\Phi$. Since $(\mathbf{V}, \Phi)$ is admissible, every $\varphi$ appears exactly once in each column of $\Phi$. Therefore, the row sums of the substitution matrix $S(\Phi)=\left(\# \Phi_{i j}\right)_{1 \leq i, j \leq m}$ are all equal. Let $q \in \mathbb{N}$ be this row sum. Thus $q$ is an eigenvalue of $S(\Phi)$, with corresponding normalized eigenvector $(1 / m, 1 / m, \ldots, 1 / m)^{T}$. Since $S(\Phi)$ is primitive, it follows from the Perron-Frobenius theorem that $q$ is the unique eigenvalue of $S(\Phi)$ which is largest in modulus (since it is the unique one with a strictly positive eigenvector). It is well known (and easy to see) that the corresponding (normalized) eigenvector contains the relative frequencies of the points of each type. Thus, all types of points have the same frequency in $\mathbf{V}$.

Assume that there is some $\Psi_{0}[a]$ such that $\# \Psi_{0}[a]=1$. This means that all points and only the points in $a+L^{\prime}$ are of the same type, which occurs with relative frequency $1 / m$. Therefore, the index $\left[L: L^{\prime}\right]$ equals $m$, but there are exactly $m$ types, and each coset $L / L^{\prime}$ contains at least one type. Then the only possibility is $\# \Psi_{0}[b]=1$ for all $b$ and $\mathbf{V}$ is periodic (with respect to the lattice $L^{\prime}$ ).

Under the nonperiodicity assumption of the corollary, it now follows that each $\Psi_{0}[a]$ contains more than one element, which completes the proof.

The following theorem allows us to extend the results to nonadmissible LSS. Under a certain condition, we can find for a primitive LSS $(\mathbf{V}, \Phi)$ an admissible one, say, $\left(\mathbf{V}^{\prime}, \Phi^{\prime}\right)$, such that $\mathbf{V}$ and $\mathbf{V}^{\prime}$ are MLD. Here and in what follows, $\mathbf{V} \cap R \mathbb{B}^{d}$ is to be read as $\left(V_{1} \cap R \mathbb{B}^{d}, \ldots, V_{m} \cap R \mathbb{B}^{d}\right)$.

Definition 4.10. Let $(\mathbf{V}, \Phi)$ be a primitive LSS and let $F$ be a fundamental domain of the lattice $\operatorname{supp}(\mathbf{V})$. We say that $(\mathbf{V}, \Phi)$ is nicely growing if there is an $R>0$ such that, for all $R$-clusters $\mathbf{V} \cap\left(t+R \mathbb{B}^{d}\right)$,

$$
\operatorname{supp}(\mathbf{V}) \cap\left(Q t+Q F+R \mathbb{B}^{d}\right) \subset \operatorname{supp}\left(\Phi\left(\mathbf{V} \cap\left(t+R \mathbb{B}^{d}\right)\right)\right) .
$$

In plain words: In particular, we require that the action of $\Phi$ maps the set of points of $\mathbf{V} \cap R \mathbb{B}^{d}$ to the points inside a region which is considerably larger, namely, $\mathbf{V} \cap(Q F+$ $R \mathbb{B}^{d}$ ) (and maybe to some more points). In general, the points of $\mathbf{V}$ inside any ball of radius $R$, centered at $t$, are mapped to the points in the set $\mathbf{V} \cap\left(Q t+Q F+R \mathbb{B}^{d}\right)$, "centered" at $Q t$ (and maybe to some more points).

Remark. All admissible substitution systems are nicely growing. If $(\mathbf{V}, \Phi)$ is admissible, then $\Phi$ maps $\mathbf{V} \cap R \mathbb{B}^{d}$ onto $\mathbf{V} \cap Q\left(R \mathbb{B}^{d}\right)$ (and possibly more), at least for $R$ large enough. (For example, the admissible substitution of the substitution system $(\mathbf{V}, \Phi)$ in Example 1 in Section 6 maps the cluster $\mathbf{V} \cap \mathbb{B}^{1}=\mathbf{V} \cap[-1,1]$ to the cluster $\mathbf{V} \cap[-6,11]$, which is a superset of $\mathbf{V} \cap([0,6]+[-1,1])=\mathbf{V} \cap[-1,7]$, thus (2) is fulfilled in this example for $t=0, R=1$.) Then the number of points in the support of the left-hand side of (2) grows approximately like $R^{d}+c R^{d-1}$, where the number of points in the support of the right-hand side grows like $R^{d}|\operatorname{det}(Q)|$. Since $|\operatorname{det}(Q)|>1$, (2) is fulfilled for $R$ large enough. This shows that all admissible substitutions are nicely growing. 
This is also true for the nonadmissible substitutions in Example 5 in Section 6. Essentially, all primitive lattice substitution systems known to the authors are nicely growing (but see Example 6 in Section 6). Note that the requirement of checking $R$ clusters is a finite problem, since there are only finitely many translation classes of them.

Theorem 4.11. Let $(\mathbf{V}, \Phi)$ be a nicely growing primitive LSS. Then there exists $\left(\mathbf{V}^{\prime}, \Phi^{\prime}\right)$, such that $\mathbf{V}$ and $\mathbf{V}^{\prime}$ are $M L D$ and $\left(\mathbf{V}^{\prime}, \Phi^{\prime}\right)$ is admissible.

Note that our general assumption on $\mathbf{V}$-all $r$-clusters in $\mathbf{V}$ are contained in some iteration $\Phi^{k}(\mathbf{v})$ of a single point $\mathbf{v}$-ensures that $\mathbf{V}$ is repetitive.

Proof. The basic idea is to construct $\mathbf{V}^{\prime}$ out of $\mathbf{V}$ by assigning to each colored point in $\mathbf{V}$ a colored point in $\mathbf{V}^{\prime}$ at the same position, where the color of the latter one depends on the local surrounding of the former one. This construction is well known, see for example [KeP] ("forcing the border") or [ORS] ("collaring"). In general, V' will use more colors than $\mathbf{V}$. Then the admissible MFS $\Phi^{\prime}$ arises in a quite natural way, using the property $\Phi(\mathbf{V})=\mathbf{V}$.

Let $R>0$ such that

$$
\operatorname{supp}(\mathbf{V}) \cap\left(Q F+R \mathbb{B}^{d}\right) \subset \operatorname{supp}\left(\Phi\left(\mathbf{V} \cap R \mathbb{B}^{d}\right)\right) .
$$

Since $(\mathbf{V}, \Phi)$ is nicely growing, we find such an $R$ by Definition 4.10 . Let $u_{1}, \ldots, u_{d}$ be a basis of the lattice $L$, i.e., $L:=\left\langle u_{1}, \ldots, u_{d}\right\rangle_{\mathbb{Z}}$. We introduce the fundamental domain

$$
F=\left\{\sum_{i=1}^{d} \lambda_{i} u_{i} \mid 0 \leq \lambda_{i}<1\right\}
$$

of $L$. For a fixed lattice $L$, the lattice basis is not unique, hence there are many possibilities how $F$ may look. For simplicity, we may choose the vectors $u_{i}$ such that the diameter $\operatorname{diam}(F)$ becomes minimal among all possible fundamental domains. However, this is not relevant in the following.

Let $x \in L$. Then any set $x+F$ contains exactly one element of $L$, namely $x$, and any set $Q x+Q F$ contains exactly $|\operatorname{det}(Q)|$ elements of $L$, which just are representatives of $L / Q L$.

Consider all types of $R$-clusters in $\mathbf{V}$, i.e., all sets

$$
\mathbf{V} \cap\left(x+R \mathbb{B}^{d}\right):=\left(V_{1} \cap\left(x+R \mathbb{B}^{d}\right), \ldots, V_{m} \cap\left(x+R \mathbb{B}^{d}\right)\right),
$$

where $x \in L$. There are finitely many translation classes of these, $\left[C^{(1)}\right],\left[C^{(2)}\right], \ldots$, $\left[C^{(n)}\right]$, say. For simplicity, we represent every translation class by a cluster $C^{(i)}$ centered at the origin. Note that the $C^{(i)}$ are of the form $\left(C_{1}^{(i)}, \ldots, C_{m}^{(i)}\right)$. Now we construct $\mathbf{V}^{\prime}$ out of $\mathbf{V}$ in the following way:

$$
V_{i}^{\prime}:=\left\{x \in L \mid \mathbf{V} \cap\left(x+R \mathbb{B}^{d}\right) \in\left[C^{(i)}\right]\right\},
$$

and $\mathbf{V}^{\prime}=\left(V_{1}^{\prime}, \ldots, V_{n}^{\prime}\right)$. In plain words: If $x$ is the center of a cluster of type $C^{(i)}$ in $\mathbf{V}$, then $x$ in $\mathbf{V}^{\prime}$ gets color $i$. Since all lattice points $x \in L$ are covered, we get $L=\bigcup_{1 \leq i \leq n} V_{i}^{\prime}$. Clearly, any $x \in L$ is the center of exactly one $R$-cluster, therefore this union is disjoint. 
The MFS $\Phi^{\prime}$ is given as follows: Let $\mathbf{V}^{\prime} \cap\{x\}$ be of type $j$, i.e., $x \in V_{j}^{\prime}$. Now define $\Phi^{\prime}$ by

$$
\Phi^{\prime}\left(\mathbf{V}^{\prime} \cap\{x\}\right):=\mathbf{V} \cap(Q x+Q F)=\left((Q x+Q F) \cap V_{1}^{\prime}, \ldots,(Q x+Q F) \cap V_{n}^{\prime}\right) .
$$

The sets $(Q x+Q F) \cap V_{i}^{\prime}$ contain finitely many points, which define the maps in $\Phi_{i j}^{\prime}$ as follows. Let $j$ be fixed. If $(Q x+Q F) \cap V_{i}^{\prime}=\left\{b_{1}, b_{2}, \ldots, b_{k}\right\}$, then set $a_{i j \ell}:=$ $b_{\ell}-Q x \in Q F \cap L, \varphi_{\ell}(y)=Q y+a_{i j \ell}(1 \leq \ell \leq k)$ and $\Phi_{i j}^{\prime}=\left\{\varphi_{1}, \varphi_{2}, \ldots, \varphi_{k}\right\}$.

We claim that $\left(\mathbf{V}^{\prime}, \Phi^{\prime}\right)$ is an admissible primitive LSS such that $\mathbf{V}$ and $\mathbf{V}^{\prime}$ are MLD. The maps of $\Phi^{\prime}$ are of the form $x \mapsto Q x+a_{i j \ell}$. By construction, the substitution given by $\Phi^{\prime}$ maps every element of $\mathbf{V}^{\prime}$ to $|\operatorname{det}(Q)|$ elements, which are contained in some translate of $Q F$. Moreover, every $a_{i j \ell}$ is an element of $Q F \cap L$, which is a fixed system of representatives of $L / Q L$. Thus the definition of admissibility is fulfilled.

Obviously, a point in $\mathbf{V}^{\prime}$ determines an $R$-cluster in $\mathbf{V}$, so $\mathbf{V}$ is locally derivable from $\mathbf{V}^{\prime}$. By construction, $\mathbf{V}^{\prime}$ is locally derivable from $\mathbf{V}$, so $\mathbf{V}$ and $\mathbf{V}^{\prime}$ are MLD.

Let $\mathbf{V} \cap\{x\}$ be of type $i, R>0$. Since $(\mathbf{V}, \Phi)$ is primitive, and by our general assumption (see the beginning of Section 3), $\Phi^{k}(\mathbf{V} \cap\{x\})$ contains all types of $R$-clusters for some appropriate $k$. Therefore, applying $\left(\Phi^{\prime}\right)^{k}$ to the corresponding point $\mathbf{V}^{\prime} \cap\{x\}$ yields all types of points in $\mathbf{V}^{\prime}$. The choice of $\mathbf{V} \cap\{x\}$ is arbitrary, so it can be the center of any type of $R$-cluster in $\mathbf{V}$. Altogether, this means that, for any $\mathbf{V}^{\prime} \cap\{x\}$, there is $k \in \mathbb{N}$ such that $\left(\Phi^{\prime}\right)^{k}\left(\mathbf{V}^{\prime} \cap\{x\}\right)$ contains all types of points that occur in $\mathbf{V}^{\prime}$. This is equivalent to $\Phi^{\prime}$ being primitive.

All we are left to show is that $\Phi^{\prime}$ is well-defined, i.e., that two points of the same type are substituted in the same way. This follows essentially from the condition $\Phi(\mathbf{V})=\mathbf{V}$ : Since two congruent clusters in $\mathbf{V}$ are mapped by $\Phi$ to congruent clusters in $\Phi(\mathbf{V})$, the corresponding points in $\mathbf{V}^{\prime}$ are mapped to congruent clusters in $\mathbf{V}^{\prime}$. Formally, we need to show: If $x, y \in V_{i}^{\prime}$ for some $i$, i.e., $\mathbf{V}^{\prime} \cap\{x\}=\left(\mathbf{V}^{\prime} \cap\{y\}\right)+x-y$, then

$$
\Phi^{\prime}\left(\mathbf{V}^{\prime} \cap\{x\}\right)=\Phi^{\prime}\left(\mathbf{V}^{\prime} \cap\{y\}\right)+Q(x-y)
$$

holds. So, let $\mathbf{V}^{\prime} \cap\{x\}=\left(\mathbf{V}^{\prime} \cap\{y\}\right)+x-y$. By construction of $\mathbf{V}^{\prime}$, it follows that the corresponding $R$-clusters in $\mathbf{V}$ are of the same type, i.e.,

$$
\mathbf{V} \cap\left(x+R \mathbb{B}^{d}\right)=\left(\mathbf{V} \cap\left(y+R \mathbb{B}^{d}\right)\right)+x-y .
$$

Since $\Phi(\mathbf{V})=\mathbf{V}$, we obtain

$$
\Phi\left(\mathbf{V} \cap\left(x+R \mathbb{B}^{d}\right)\right)=\Phi\left(\left(\mathbf{V} \cap\left(y+R \mathbb{B}^{d}\right)\right)+x-y\right)=\Phi\left(\mathbf{V} \cap\left(y+R \mathbb{B}^{d}\right)\right)+Q(x-y) .
$$

Consider $\mathbf{V} \cap\left(Q x+Q F+R \mathbb{B}^{d}\right), \mathbf{V} \cap\left(Q y+Q F+R \mathbb{B}^{d}\right)+Q(x-y)$. The growing nicely condition implies

$$
\begin{gathered}
\operatorname{supp}\left(\mathbf{V} \cap\left(Q x+Q F+R \mathbb{B}^{d}\right)\right) \subset \operatorname{supp}\left(\Phi\left(\mathbf{V} \cap\left(x+R \mathbb{B}^{d}\right)\right)\right), \\
\| \\
\operatorname{supp}\left(\mathbf{V} \cap\left(Q y+Q F+R \mathbb{B}^{d}\right)\right)+Q(x-y) \subset \operatorname{supp}\left(\Phi\left(\mathbf{V} \cap\left(y+R \mathbb{B}^{d}\right)\right)\right)+Q(x-y) .
\end{gathered}
$$


The four sets $\mathbf{V} \cap\left(Q x+Q F+R \mathbb{B}^{d}\right), \Phi\left(\mathbf{V} \cap\left(x+R \mathbb{B}^{d}\right)\right), \mathbf{V} \cap\left(Q y+Q F+R \mathbb{B}^{d}\right)$ and $\Phi\left(\mathbf{V} \cap\left(y+R \mathbb{B}^{d}\right)\right)$ occurring in the above equation are all subsets of $\mathbf{V}$ as multicomponent sets, i.e., with respect to colors. We obtain

$$
\begin{gathered}
\mathbf{V} \cap\left(Q x+Q F+R \mathbb{B}^{d}\right) \subset \Phi\left(\mathbf{V} \cap\left(x+R \mathbb{B}^{d}\right)\right) \\
\| \\
\mathbf{V} \cap\left(Q y+Q F+R \mathbb{B}^{d}\right)+Q(x-y) \subset \Phi\left(\mathbf{V} \cap\left(y+R \mathbb{B}^{d}\right)\right)+Q(x-y) .
\end{gathered}
$$

Obviously, the supports of the left-hand sides are equal. Therefore, it follows from the above equation, that the left-hand sides are equal as multi-component sets:

$$
\mathbf{V} \cap\left(Q x+Q F+R \mathbb{B}^{d}\right)=\mathbf{V} \cap\left(Q y+Q F+R \mathbb{B}^{d}\right)+Q(x-y) .
$$

By the construction of $\mathbf{V}^{\prime}$, the $R$-cluster $C_{R}=\mathbf{V} \cap\left(x+R \mathbb{B}^{d}\right)$ in $\mathbf{V}$ determines the color of $x \in \mathbf{V}^{\prime}$. Therefore, the above equation yields

$$
\mathbf{V}^{\prime} \cap(Q x+Q F)=\mathbf{V}^{\prime} \cap(Q y+Q F)+Q(x-y),
$$

and by the construction of $\Phi^{\prime}$, this is equivalent to (4). Altogether, $\left(\mathbf{V}^{\prime}, \Phi^{\prime}\right)$ is an admissible primitive LSS, such that $\mathbf{V}$ and $\mathbf{V}^{\prime}$ are MLD.

The last result proves useful as follows.

Theorem 4.12. Let $(\mathbf{V}, \Phi)$ be a primitive LSS, and let $\left(\mathbf{V}^{\prime}, \Phi^{\prime}\right)$ be an admissible one, derived from $(\mathbf{V}, \Phi)$ as in the proof of Theorem 4.11. If $\mathbf{V}^{\prime}$ consists of model sets, then so does $\mathbf{V}$.

Proof. Let $\mathbf{V}=\left(V_{1}, \ldots, V_{m}\right)$. By the construction of $\mathbf{V}^{\prime}$, each set $V_{i}$ is partitioned into sets $V_{i_{1}}^{\prime}, V_{i_{2}}^{\prime}, \ldots, V_{i_{k}}^{\prime}$ belonging to $\mathbf{V}^{\prime}$. Recall: An element of $\mathbf{V}^{\prime}$ at position $x$ got its type according to the $R$-cluster surrounding $x$ in $\mathbf{V}$. Denote all translation classes of $R$-clusters with center of type $i$ by $\left[C_{i_{1}}\right],\left[C_{i_{2}}\right], \ldots,\left[C_{i_{k}}\right]$. Then $V_{i}=V_{i_{1}}^{\prime} \cup V_{i_{2}}^{\prime} \cup \cdots \cup V_{i_{k}}^{\prime}$.

Let $\mathbf{V}^{\prime}$ consist of model sets. This means that each $V_{i_{j}}^{\prime}$ is a model set. To each $V_{i_{j}}^{\prime}$ belongs a window set $W_{i_{j}}^{\prime}$. Let $V_{i_{1}}^{\prime}, V_{i_{2}}^{\prime}, \ldots, V_{i_{k}}^{\prime}$ be the sets assigned to $V_{i}$, i.e., $V_{i}=\bigcup_{1 \leq j \leq k} V_{i_{j}}^{\prime}$. Let $\Omega_{i_{j}}^{\prime}$ be the window of $V_{i_{j}}^{\prime}$ (see Definition 3.1). Then

$$
V_{i}=\bigcup_{1 \leq j \leq k} V_{i_{j}}^{\prime}=\bigcup_{1 \leq j \leq k} \Lambda\left(\Omega_{i_{j}}^{\prime}\right)=\Lambda\left(\bigcup_{1 \leq j \leq k} \Omega_{i_{j}}^{\prime}\right) .
$$

Since the windows $\Omega_{i_{j}}^{\prime}$ have nonempty interior and compact closure, this is also true for their (finite) union. Thus $V_{i}$ is a model set, too.

\section{Remarks on Complexity}

Theorem 4.5 gives an exponential upper bound for computing modular coincidences. This may be far away from the best upper bound. In particular, it is not known whether the required number of iterations is polynomial in $m$ or not. 
If $k=2^{m}$, the $k$ th power of an $(m \times m)$-MFS $\Phi$ can be computed by $m-1$ "matrix multiplications," to be understood as stated after Definition 2.1. If $k^{\prime}<k$ and $\Phi^{k^{\prime}}$ already admits a modular coincidence, then $\Phi^{k}$ also does. Therefore, it suffices to compute $\Phi, \Phi^{2}, \Phi^{4}, \Phi^{8} \ldots$. At first sight, this would lead to a polynomial bound for computing modular coincidences. However, unlike usual matrix multiplications, the costs for each multiplication is not $m^{3}$, but the costs per multiplication increase with $k$. We do not study this question in detail here, but, essentially, the costs grow faster than the sum of the entries of the substitution matrix $S\left(\Phi^{k}\right)$.

The authors conjecture that the lowest upper bound is quadratic in $m$, hence not linear in $m$. Consider the following example:

$$
1 \mapsto 12, \quad 2 \mapsto 23, \quad 3 \mapsto 34, \quad \ldots, \quad m-1 \mapsto m-1 m, \quad m \mapsto 11 .
$$

If $m=2$ this reads as the well-known substitution $a \rightarrow a b, b \rightarrow a a$. For $2 \leq m \leq 16$, the first modular coincidence occurs in $\Phi^{(m-1)^{2}}$. This is a hint that a general upper bound is quadratic in $m$. It will be difficult to find a worse example, since the dimension $(=1)$ and the factor $\operatorname{det}(Q)(=2)$ are the smallest possible for an admissible primitive LSS, as well as the number of pairwise coincidences $(=1)$. For $m \in\{2,3,4,5,6\}$, we computed for all substitutions with these three properties the minimal number $k$, such that $\Phi^{k}$ shows a modular coincidence (if there is such a $k$ ). It turned out that the substitution (5) yields the maximum value of $k$ in these cases.

\section{Examples of Sequences and Tilings}

1. Periodic Substitution I. The primitive substitution given by the following MFS yields a periodic sequence. Here $h_{i}$ denotes the map $h_{i}(x)=6 x+i, i \in \mathbb{Z}$.

$$
\Phi=\left(\begin{array}{lll}
\left\{h_{0}, h_{3}\right\} & \left\{h_{0}, h_{3}\right\} & \left\{h_{0}, h_{3}\right\} \\
\left\{h_{1}, h_{5}\right\} & \left\{h_{1}, h_{5}\right\} & \left\{h_{1}, h_{5}\right\} \\
\left\{h_{2}, h_{4}\right\} & \left\{h_{2}, h_{4}\right\} & \left\{h_{2}, h_{4}\right\}
\end{array}\right) .
$$

In symbolic notation, this reads $a \mapsto a b c a c b, b \mapsto a b c a c b, c \mapsto a b c a c b$. This substitution on $L=\mathbb{Z}$ yields $L_{a}=3 \mathbb{Z}, L_{b}=2 \mathbb{Z}$ and $L_{c}=2 \mathbb{Z}$ (and therefore $L^{\prime}=L$ ). Note that here-as in some of the following examples-we use letters $a, b, c$ instead of numbers $1,2,3$. To obtain a tiling, replace the letter at position $n$ by the interval $[n, n+1]$ (and label it with its letter). To obtain a multi-component Delone set, let $\mathbf{V}=\left(V_{a}, V_{b}, V_{c}\right)$, where $V_{a}$ contains all positions of letters $a$, etc.

2. Periodic Substitution II. The trivial substitution $a \mapsto a b, b \mapsto a b$ on $L=\mathbb{Z}$ yields the periodic solution ... ababababababa .. (and therefore $V_{a}=2 \mathbb{Z}, V_{b}=2 \mathbb{Z}+1$ and $L^{\prime}=2 \mathbb{Z}$ ). It already shows a modular coincidence relative to $Q L^{\prime}$ (each $\Phi[i], 0 \leq i \leq 3$, consists of exactly one element). Following the construction preceding Definition 3.2, one would obtain as internal space $H=C_{2} \times \mathbb{Z}_{2}$ (where $C_{2}=\mathbb{Z} / 2 \mathbb{Z}$ is the cyclic group of order 2 , and $\mathbb{Z}_{2}$ are the 2 -adic integers $)$ and the lattice $\tilde{L}=\left\{(t, t \bmod 2, t) \in \mathbb{R} \times C_{2} \times \mathbb{Z}_{2} \mid\right.$ $t \in \mathbb{Z}\}$, but it is easy to see that $H^{\prime}=C_{2}$ and $\tilde{L}^{\prime}=\left\{(t, t \bmod 2) \in \mathbb{R} \times C_{2} \mid t \in \mathbb{Z}\right\}$ suffices. 
3. Thue-Morse Sequence. The Thue-Morse sequence is defined as the fixed point beginning by 0 of the substitution $0 \mapsto 01,1 \mapsto 10$ (it starts $0110100110010110100 \ldots$ ). There is also an arithmetic definition of this sequence (e.g., see [H, p. 2] and [PF, 2.1.1]): For any integer $n$, denote by $S_{2}(n)$ the sum of the dyadic (binary) digits of $n$, i.e., $S_{2}(n)=\sum_{i \geq 0} n_{i}$, if $n=\sum_{i \geq 0} n_{i} 2^{i}\left(n_{i} \in\{0,1\}\right)$. Then the $n$th symbol in the sequence is a 1 iff $S_{2}(n)$ is odd. Obviously, for $n<2^{k}$, we have the following: if $S_{2}(n)$ is odd, then $S_{2}\left(n+2^{k}\right)$ is even and vice versa. Therefore $\Psi_{k}[n]=\left\{i \mid\left(n+2^{k} \mathbb{Z}\right) \cap V_{i}\right\}=\{0,1\}$ for all $k$ and $n$, and the Thue-Morse substitution admits no modular coincidence.

4. Parents and Children. Consider a substitution defined by $a \mapsto a b a, b \mapsto b c c$ and $c \mapsto a b c$ (this substitution arises in the study of the Kolakoski sequence over the alphabet $\{2,4\}$, see [Si]). The corresponding MFS $\Phi$ is given by

$$
\Phi=\left(\begin{array}{ccc}
\left\{h_{0}, h_{2}\right\} & \varnothing & \left\{h_{0}\right\} \\
\left\{h_{1}\right\} & \left\{h_{0}\right\} & \left\{h_{1}\right\} \\
\varnothing & \left\{h_{1}, h_{2}\right\} & \left\{h_{2}\right\}
\end{array}\right),
$$

with $h_{i}(x)=3 x+i$. A fixed point is given by . . aba bcc abc $\dot{a} b a b c c a b a \ldots$, where the overdot marks the zeroth position. It is easy to check that here we have $L=\mathbb{Z}=L^{\prime}$. In this case, as $L=L^{\prime}$ and $\Phi$ is admissible, the check for modular coincidence reduces to finding a map $g$ which is shows up in only one row of $\Phi^{k}$ for some $k \in \mathbb{N}$. Therefore we get (note that $\Phi_{a a}=\left\{h_{0}, h_{2}\right\}, \Phi_{b a}=\left\{h_{1}\right\}$, etc.)

$$
\Phi[0] \subset \Phi_{a a} \cup \Phi_{b b} \cup \Phi_{a c}, \quad \Phi[1] \subset \Phi_{b a} \cup \Phi_{c b} \cup \Phi_{b c}, \quad \Phi[2] \subset \Phi_{a a} \cup \Phi_{c b} \cup \Phi_{c c} .
$$

Here we use letters rather than numbers to avoid confusion, because numbers are used to represent cosets below. Since none of the sets $\Phi[\cdot]$ is contained in just one row of the MFS, $\Phi$ itself does not show a modular coincidence. (For instance, $\Phi[0]$ is contained in the first and second row of $\Phi, \Phi[1]$ in the second and last row, etc.) Nevertheless, $\Phi^{2}$ shows a modular coincidence already, or more precisely, three of them:

$$
\Phi^{2}=\left(\begin{array}{ccc}
\left\{h_{0}^{2}, h_{0} h_{2}, h_{2} h_{0}, h_{2}^{2}\right\} & \left\{h_{0} h_{1}, h_{0} h_{2}\right\} & \left\{h_{0}^{2}, h_{2} h_{0}, h_{0} h_{2}\right\} \\
\left\{h_{1} h_{0}, h_{1} h_{2}, h_{0} h_{1}\right\} & \left\{h_{0}^{2}, h_{1}^{2}, h_{1} h_{2}\right\} & \left\{h_{1} h_{0}, h_{0} h_{1}, h_{1} h_{2}\right\} \\
\left\{h_{1}^{2}, h_{2} h_{1}\right\} & \left\{h_{1} h_{0}, h_{2} h_{0}, h_{2} h_{1}, h_{1}^{2}\right\} & \left\{h_{1}^{2}, h_{2} h_{1}, h_{2}^{2}\right\}
\end{array}\right) .
$$

The map $h_{0} \circ h_{2}(x)=9 x+6$ is contained in just the first row, the map $h_{1} \circ h_{2}(x)=9 x+7$ just in the second row and the map $h_{2} \circ h_{1}(x)=9 x+5$ just in the third row.

Since $L=L^{\prime}$, we have $\Psi_{0}[0]=\{a, b, c\}$. The next step is easy: For $\Psi_{1}[i]$ we just have to consider the maps $\Phi[i]$ (see the first indices of the matrix elements of $\Phi$ involved in $\Phi[i])$ to obtain

$$
\Psi_{1}[0]=\{a, b\}, \quad \Psi_{1}[1]=\{b, c\}, \quad \Psi_{1}[2]=\{a, c\} .
$$

Basically, we make use of the (unique) ternary expansion of each natural number and that the maps of the MFS line up with this ternary expansion. For example, to calculate $\Psi_{2}$ [7], we observe that $7=2 \cdot 3+1$, therefore $\Psi_{2}[7]$ is determined by the $i$ 's of $\Phi_{i j}[1]$ 
with $j \in \Psi_{1}[2]$, yielding $\Psi_{2}[7]=\{b\}$. For completeness, we list all $\Psi_{2}$ :

$$
\begin{aligned}
& \Psi_{2}[0]=\{a, b\}, \quad \Psi_{2}[1]=\{b, c\}, \quad \Psi_{2}[2]=\{a, c\}, \quad \Psi_{2}[3]=\{a, b\}, \\
& \Psi_{2}[4]=\{b, c\}, \quad \Psi_{2}[5]=\{c\}, \quad \Psi_{2}[6]=\{a\}, \quad \Psi_{2}[7]=\{b\}, \quad \Psi_{2}[8]=\{a, c\} .
\end{aligned}
$$

Here, the children of $\Psi_{1}[i]$ are $\Psi_{2}[3 \cdot i], \Psi_{2}[3 \cdot i+1]$ and $\Psi_{2}[3 \cdot i+2]$. Since $\Psi_{2}[5]$, $\Psi_{2}[6]$ and $\Psi_{2}[7]$ are singleton sets, we have a modular coincidence.

Note the correspondence between $\Phi^{k}[i]$ and $\Psi_{k}[j]$. For instance, the fact that $\Psi_{2}[5]=$ $\{c\}$ corresponds to the fact that $h_{2} \circ h_{1}(x)=9 x+5$ is contained in the last (the "cth") row of $\Phi^{2}$.

5. Nonadmissible Substitution I. Consider the one-dimensional substitution given by the following MFS (where $h_{i}$ denotes the map $h_{i}(x)=3 x+i$ ):

$$
\Phi=\left(\begin{array}{ccc}
\left\{h_{0}\right\} & \left\{h_{1}\right\} & \left\{h_{2}\right\} \\
\left\{h_{1}, h_{5}\right\} & \left\{h_{0}\right\} & \left\{h_{1}, h_{5}\right\} \\
\left\{h_{2}\right\} & \varnothing & \left\{h_{0}\right\}
\end{array}\right) .
$$

In symbolic notation, this corresponds to the substitution

$$
a \rightarrow a b c_{-} b, \quad b \rightarrow b a, \quad c \rightarrow c b a_{-} b,
$$

where _ stands for an empty position. A fixed point of this substitution is

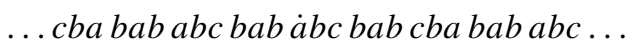

The overdot marks the zeroth position. Obviously, this substitution is not admissible. Nevertheless, a brief look at the first iterations $\Phi^{k}(\{0\}, \varnothing, \varnothing)$ shows that this substitution is nicely growing. It is easy to see that this substitution is equivalent to the admissible substitution

$$
a \rightarrow a b c, \quad b \rightarrow b a b, \quad c \rightarrow c b a .
$$

Both substitutions yield the same sequences, therefore the sequences are clearly MLD. Here we did not use the construction of Theorem 4.12. This would yield another sequence $\mathbf{V}^{\prime}$ MLD to (6), but it would use more than three letters. Namely, the construction works with $R=2$. There are eight different types of 2-clusters (five-letter words) in (6), yielding eight different letters in the new sequence $\mathbf{V}^{\prime}$. This example is easily generalized to more than one dimension.

6. Nonadmissible Substitution II. The following MFS yields another nonadmissible substitution:

$$
\Phi=\left(\begin{array}{cccc}
\left\{h_{15}\right\} & \left\{h_{15}\right\} & \varnothing & \left\{h_{15}\right\} \\
\left\{h_{14}\right\} & \left\{h_{13}\right\} & \left\{h_{15}\right\} & \left\{h_{14}, h_{13}\right\} \\
\left\{h_{13}\right\} & \left\{h_{14}\right\} & \left\{h_{12}, h_{14}, h_{13}\right\} & \varnothing \\
\left\{h_{12}, h_{0}\right\} & \left\{h_{12}\right\} & \varnothing & \varnothing
\end{array}\right),
$$



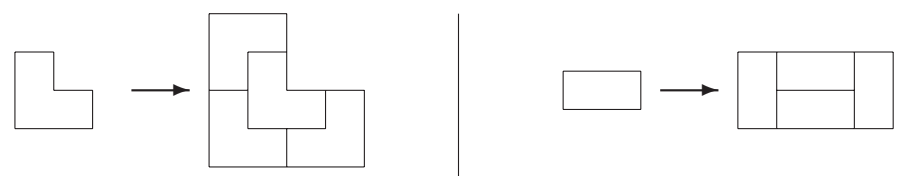

Fig. 1. Tile substitution for the chair tiling (left) and for the table tiling (right).

where $h_{i}(x)=4 x+i$. In symbolic notation, this would look quite weird, since the action of $\Phi$ contains large shifts to the right. For example, a letter $a$ at position 0 becomes a word $d_{-} \ldots{ }_{-} d c b a$ under the action of $\Phi$, showing a large gap between the first letter $d$ at position 0 and the second letter $d$ at position 12. This substitution is clearly not admissible. Nevertheless, there is a $\mathbf{V}$ such that $\Phi(\mathbf{V})=\mathbf{V}$. For example, let $C=(\{-5\}, \varnothing,\{-4\}, \varnothing)$. The iteration of $\Phi$ on $C$ yields a sequence with $\Phi(\mathbf{V})=\mathbf{V}$, namely $\mathbf{V}=\bigcup_{k \in \mathbb{N}} \Phi^{k}(C)$. Thus $(\mathbf{V}, \Phi)$ is a primitive LSS. It is not easy to see whether this substitution is nicely growing. If so, it can be shown that $R$ must be at least 15. (Note that the difference between the smallest and the largest translational part occurring in $\Phi$ is 15 .)

This example can be generalized to higher dimensions, too. It can also be generalized to any number $m \geq 4$ of types of points such that the difference between the smallest and the largest translational part occurring in $\Phi$ is $m^{2}-1$ (and we really have an LSS). In this case it can be shown that $R$ must be at least $m^{2}-1$, if the corresponding substitution system is nicely growing. Anyway, the substitution above on four letters yields the same sequences as the admissible substitution

$$
a \rightarrow d c b a, \quad b \rightarrow d b c a, \quad c \rightarrow c c c b, \quad d \rightarrow d b b a,
$$

showing a modular coincidence in $\Phi^{2}$.

7. The Chair Tiling and the Table Tiling. The chair tiling and the table tiling (also called the domino tiling) and their spectral properties are discussed in detail in [So], $[\mathrm{R}]$ and $[\mathrm{BMS}]$. They are generated by the tile substitutions in Fig. 1. The expansion $Q$ acts as scaling by a factor 2 , and the rotated versions of the shown tiles are substituted analogously. Both tile substitutions can be described by substitutions over the alphabet $\{p, q, r, s\}$ on $L=\mathbb{Z}^{2}$, where one uses the labeling of Fig. 2 .

These labelings yield the (induced) substitutions in Fig. 3. Therefore, we obtain the coincidence graphs shown in Fig. 4 (observing that $L=L^{\prime}=\mathbb{Z}^{2}$ ). Note that the substitution for the table tiling admits no pairwise coincidence, therefore Corollary 4.7 yields that the induced LSS does not consist of model sets. The substitution is also a bijective substitution, so Corollary 4.9 applies, too. These graphs show that the chair

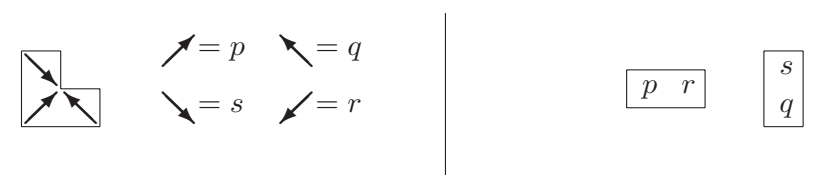

Fig. 2. Labeling for chair tiling (left) and for the table tiling (right). 


$$
\begin{aligned}
& p \mapsto \begin{array}{cc}
s & p \\
p & q
\end{array} \quad q \mapsto \begin{array}{cc}
q & r \\
p & q
\end{array} \quad p \mapsto \begin{array}{ccc}
s & p \\
q & p
\end{array} \quad q \mapsto \begin{array}{ll}
q & q \\
p & r
\end{array}
\end{aligned}
$$

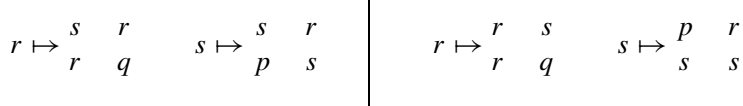

Fig. 3. Induced substitution for the chair tiling (left) and for the table tiling (right).

tiling is pure point diffractive (compare with Theorem 8.1 of [R], see also Example 7.1 of [So]), while the table tiling is not (compare with Theorem 8.2 of [R], see also Example 7.3 of [So]). We note that the internal space for the chair tiling is $\mathbb{Z}_{2}^{2}$ (which lines up with the inflation by a factor of 2 , i.e., $\mathbb{Z}_{2}^{2}=\lim _{\leftarrow k} \mathbb{Z}^{2} / 2^{k} \cdot \mathbb{Z}^{2}$ ).

8. (R1)-(R2) Tilings. In [Fra1] a family of substitutions is considered which fulfil two conditions, namely, (R1) and (R2). In our notation, these read as follows. Let $(\mathbf{V}, \Phi)$ be an admissible primitive LSS supported on $L=\mathbb{Z}^{d}, \mathbf{V}=\left(V_{1}, \ldots, V_{2 m}\right),\left[L: L^{\prime}\right]=m$.

(R1) $\Psi_{0}\left[a_{i}\right]=\{i, i+m\}(1 \leq i \leq m)$, where $a_{i}$ runs through $L / L^{\prime}$.

(R2) $\Phi_{j, i} \cap \Phi_{j, i+m}=\varnothing$ for all $i, j$.

The first condition tells us that no $\Psi_{0}[a]$ is a singleton set: each one contains two elements $i, i+m$. Therefore, the second condition tells us that there is no pairwise coincidence in any $\Psi_{0}[a]$. Thus, it follows from Corollary 4.7 that none of the multi-component Delone sets (or tilings) induced by these substitutions consist of model sets.

9. The Semi-Detached House Tiling. The tile substitution in Fig. 5 (with expansion factor 2) appears as an unsolved problem in Abbildung $17 \mathrm{of} \mathrm{[F].} \mathrm{As} \mathrm{above,} \mathrm{rotated} \mathrm{and}$ reflected versions of the tiles are substituted analogously to the shown tiles. Each of the

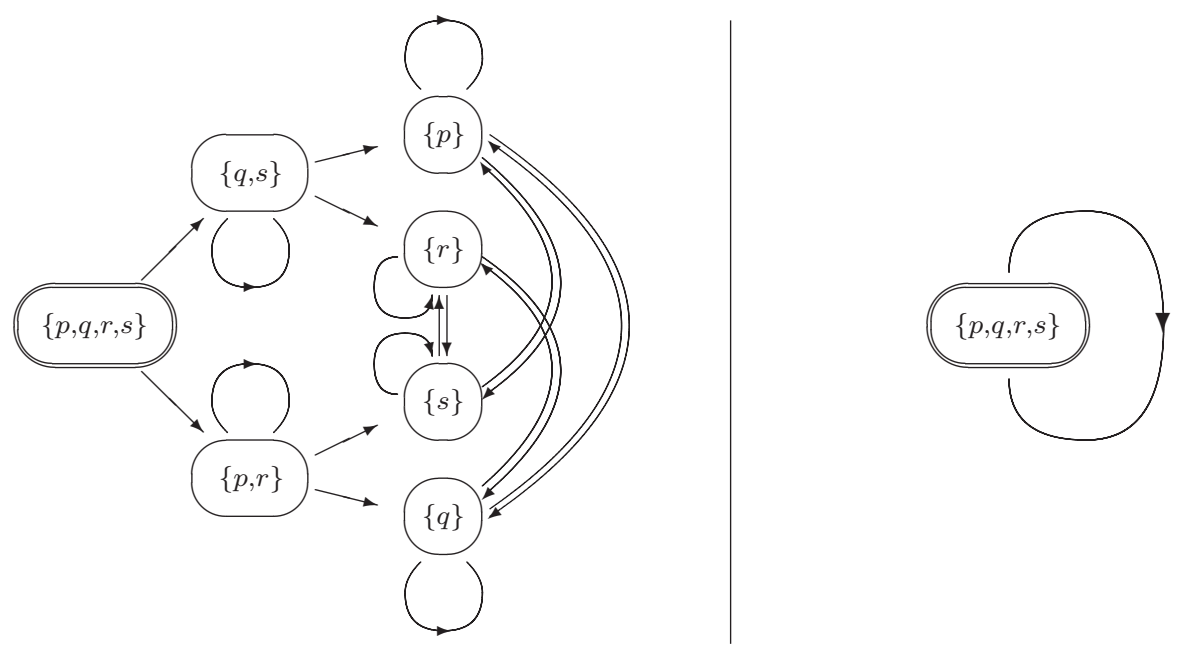

Fig. 4. Coincidence graph for the chair tiling (left) and for the table tiling (right). 

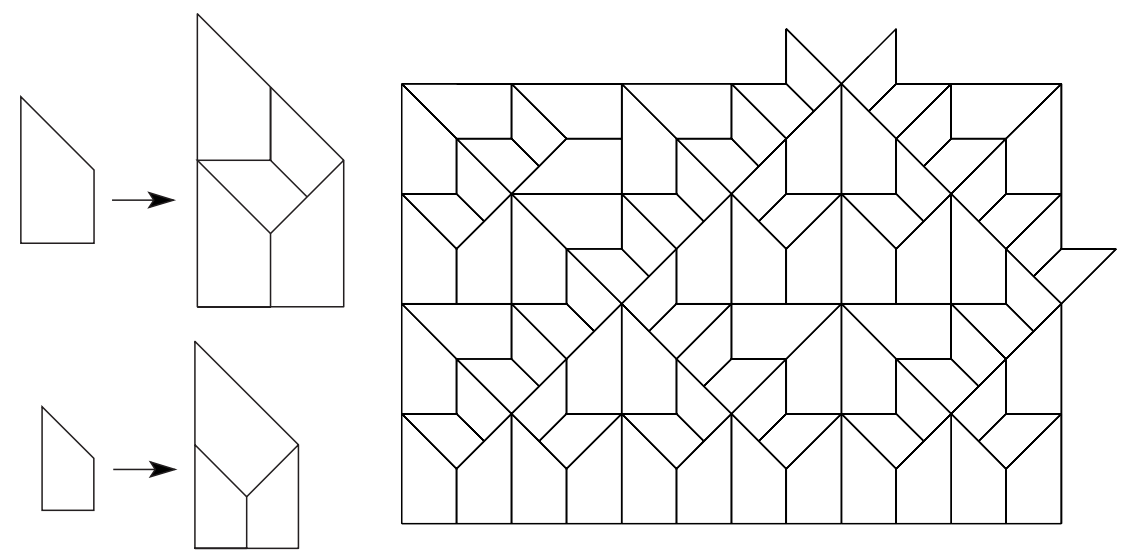

Fig. 5. The substitution for the semi-detached house tiling (left) and a part of the tiling, which (hopefully) illustrates its name.

two semi-detached houses (i.e., the big and the small one, see tile substitution in Fig. 5) arises in four different orientations and its reflected versions. To derive a substitution, we use the labeling in Fig. 6.

We then obtain the substitution for "squares" in Fig. 7, which is again a substitution on $L=\mathbb{Z}^{2}$ by a factor of 2 . Instead of using all 56 squares which occur in this substitution, we form 20 classes which are already present in Fig. 7 (i.e., squares which yield the same substitute are in the same class). We now look at the substitution induced on these classes, e.g.,

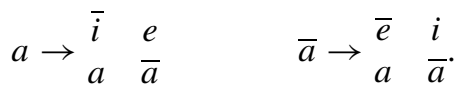

Obviously, if we do not have a modular coincidence for this induced substitution, then there is no coincidence for the original substitution either. The coincidence graph for the induced substitution is shown in Fig. 8. As suggested by Fig. $7, L^{\prime}$ forms a checkerboard with respect to $L$, i.e., $\left[L: L^{\prime}\right]=2$.

As we do not have a modular coincidence for this substitution (no vertex in the coincidence graph represents a singleton), the semi-detached house tiling does not arise

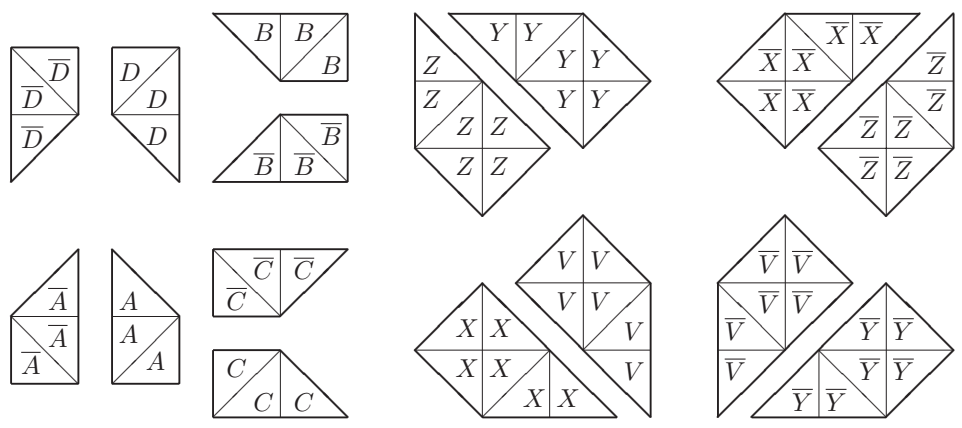

Fig. 6. Labeling of the semi-detached house tiling. 


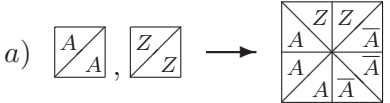

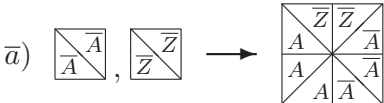
b) $B / B, Y / Y \rightarrow Y_{Y}^{B} \rightarrow \frac{B}{B}$

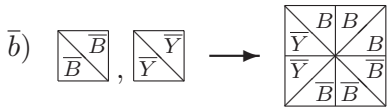

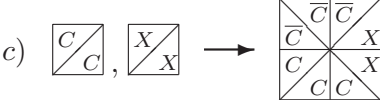

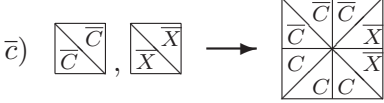
d) $V / D, V / V \rightarrow \frac{D}{D} \int_{V}^{\bar{D}} \frac{D}{D}$

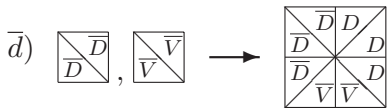
e) $Z / \bar{A}, Z_{\bar{Z}}^{Z}, Z_{\bar{Z}}^{Y} \rightarrow \frac{Y{ }^{Z}}{Y Z / \bar{Z}}$
$\bar{e}){ }_{A}^{\bar{Z}}, Z_{Z}^{\bar{Z}}, Z_{Z}^{\bar{X}} \rightarrow \frac{Z{ }^{\bar{Z}} \overline{\bar{Z}} / \bar{X}}{Z Z_{Z}{ }_{Z} \overline{\bar{X}}}$

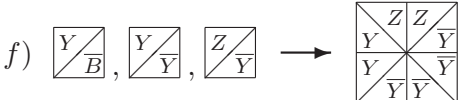

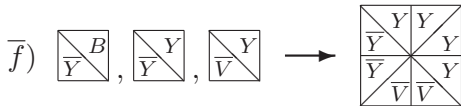
g) $\overline{\bar{C}} / \mathrm{X}, \overline{\bar{X}} / \mathrm{X}, \overline{\bar{X}} / V \rightarrow \frac{\bar{X} \bar{X} \bar{X} / x}{\bar{X} / V V_{V}^{X}}$
$\bar{g}){ }_{C} \bar{X},{ }_{X}^{\bar{X}},{ }_{X}^{\bar{Z}} \rightarrow \frac{X^{\bar{Z}} \bar{Z} / \bar{X}}{X} /_{X}^{\bar{X}}$
h) $\overline{\bar{D}} / V, \overline{\bar{V}} / V, \overline{\bar{V}} / x \rightarrow \frac{\bar{V} \bar{V} \bar{V} / x}{\bar{V} / V / V}$

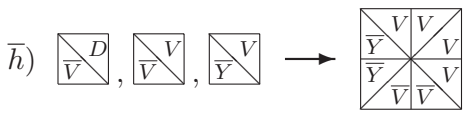

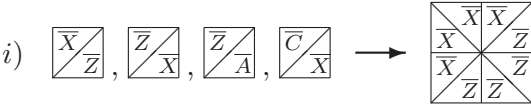

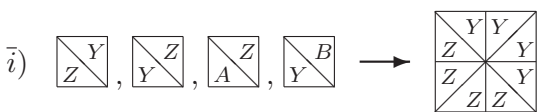

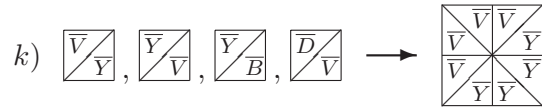

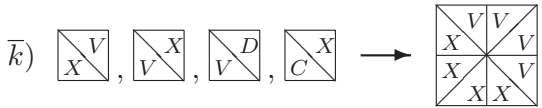

Fig. 7. Substitution on squares, derived from the substitution in Fig. 5.

from a model set and is not pure point diffractive. This nontrivial example shows that analyzing LSS and tilings can often be quite elaborate.

\section{Appendix A. Modular Coincidence versus Dekking Coincidence}

An admissible LSS in one dimension (i.e., on $L=\mathbb{Z}$ ) is known as a primitive substitution of constant length $q$, see [D], [Q] and [PF]. Here the mappings of the MFS $\Phi$ are of the form $x \mapsto q \cdot x+a_{i j k}$ where $a_{i j k} \in\{0, \ldots, q-1\}$, i.e., each letter is substituted by exactly $q$ letters.

The first step in analyzing such a substitution is to determine $L^{\prime}$. Since $L=\mathbb{Z}$, we have $L^{\prime}=r \cdot \mathbb{Z}$ and $r \in \mathbb{N}$ is to be determined. To this end, we define, for a sequence 


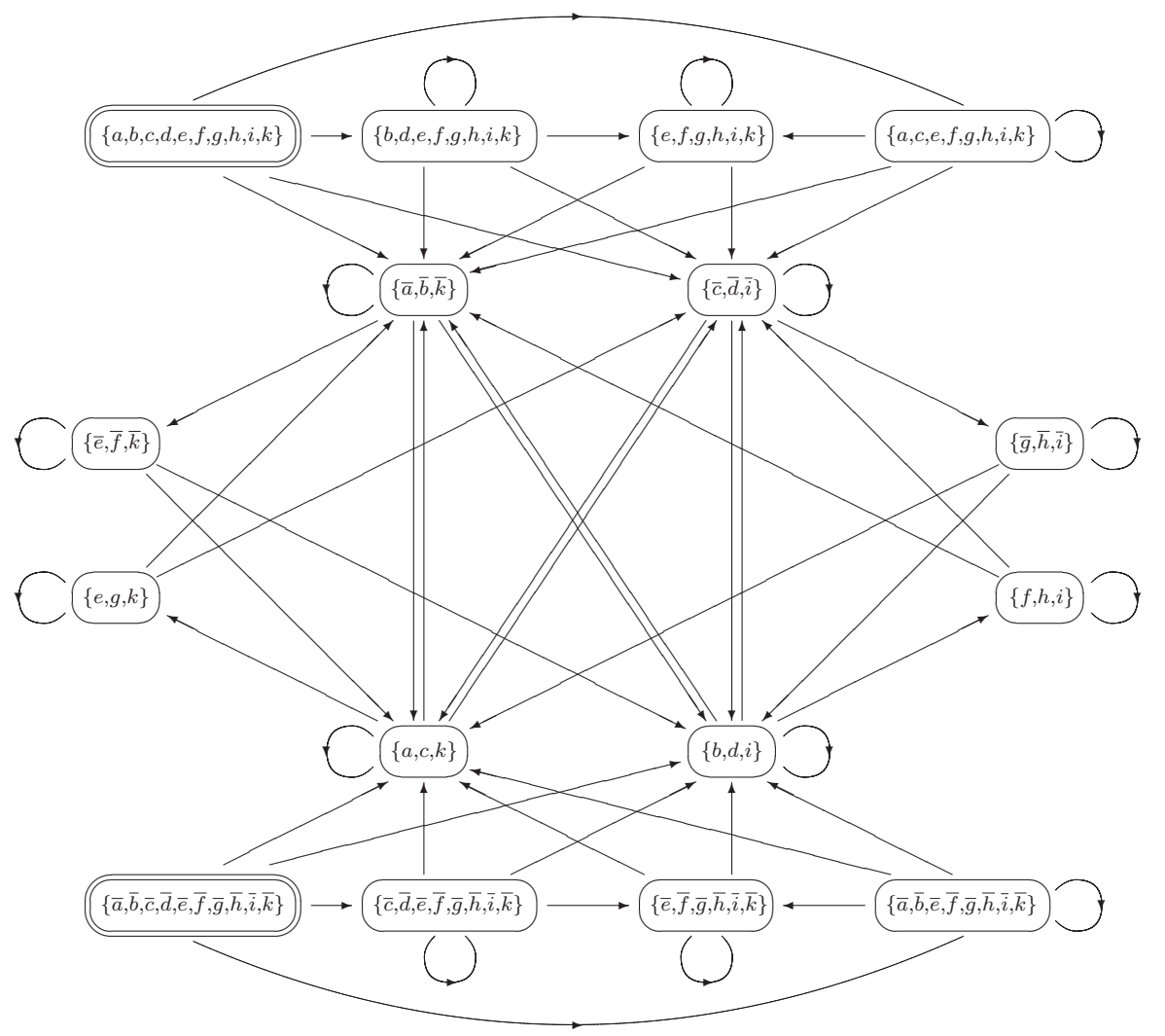

Fig. 8. The coincidence graph of the derived tiling of the semi-detached house tiling.

$u=\cdots u_{-2} u_{-1} u_{0} u_{1} u_{2} \ldots$, the sets $S_{k}=\left\{j \in \mathbb{Z} \mid u_{j+k}=u_{k}\right\}$ and numbers $g_{k}=\operatorname{gcd} S_{k}$. It is easy to see that $g_{k}=g_{k^{\prime}}$ if $u_{k}=u_{k^{\prime}}$, so that we only have to determine the $g_{k}$ once for each letter of the alphabet. Furthermore, we have $L_{i}=g_{k} \cdot \mathbb{Z}$ if $u_{k}=i$ (in other words, if $u_{k}$ is of type $i$ ) and, by $L^{\prime}=L_{1}+\cdots+L_{m}$ ( $m$ is the cardinality of the alphabet), also $r=\operatorname{gcd}\left\{g_{k} \mid k \in \mathbb{Z}\right\}$.

Let us introduce the height $h$ of a substitution of constant length $q$ (see Definition II.8 of [D] and Definition VI.1. of [Q]): $h=\max \left\{n \geq 1 \mid \operatorname{gcd}\{n, q\}=1\right.$ and $n$ divides $\left.g_{0}\right\}$. We have the following property for every $k \in \mathbb{Z}$ [D, Remark II.9(i)], [Q, VI.2.1.1.]:

$\left\{n \geq 1 \mid \operatorname{gcd}\{n, q\}=1\right.$ and $n$ divides $\left.g_{0}\right\}=\left\{n \geq 1 \mid \operatorname{gcd}\{n, q\}=1\right.$ and $n$ divides $\left.g_{k}\right\}$

From this, it follows directly that $1 \leq h \leq r \leq m$ and $h$ divides $r$, but is relatively prime to $q$. If $r=m$, then the sequence under consideration is periodic (compare with the argument in the proof of Corollary 4.9). It can happen that $h<r$.

Example. The paperfolding sequence can be generated by the substitution $a \mapsto a b$, $b \mapsto c b, c \mapsto a d, d \mapsto c d$ and a subsequent projection $\{a, b\} \mapsto 0,\{c, d\} \mapsto 1$ on a 
two-letter alphabet $\{0,1\}$. We consider the original substitution on $\{a, b, c, d\}$ for which we have $q=2, g_{k}=2$ for all $k \in \mathbb{Z}, r=2$ and $h=1$.

For a substitution $\sigma$ of constant length with $h>1$, it is always possible to introduce a substitution $\eta$ on a (usually bigger) alphabet of the same constant length with height 1 such that the sequence generated by $\sigma$ can also be obtained from the sequence generated by $\eta$, see Lemma II.17 and Definition II.20 of [D]. We illustrate the general method by an example, also compare this construction with Theorem 4.11.

Example. Consider the substitution (see the example following Definition II.20 of [D]) $0 \mapsto 010,1 \mapsto 102$ and $2 \mapsto 201$ of height 2 . This substitution generates a sequence $u=\ldots 010102010102 \ldots$. Now we take as the new alphabet all words in $\left\{u_{n \cdot h} \ldots u_{n \cdot h+h-1} \mid n \in \mathbb{Z}\right\}$, in this case just $a=01$ and $b=02$. Then the substitution $\eta$ of height 1 is given by $a \mapsto a a b$ and $b \mapsto a b a$ (since $a=01 \mapsto 010102=a a b$ ).

Let $\eta$ be a substitution of constant length $q$ and height $h=1$. We say $\eta$ admits a coincidence (or Dekking coincidence) if there exist $k$ and $0 \leq j<q^{k}$ such that the $j$ th symbol of $\eta^{k}(i)$ is the same for all letters $i$ [Q, Definition VI.3] (see also Defnition III.1 of [D]). In the above notation, $\eta$ admits a coincidence if $\eta^{k}(i)_{j}$ is the same for all $i$. A substitution $\sigma$ of constant length $q$ and height $h>1$ is said to admit a coincidence if its associated substitution of height $h=1$ admits one.

Reformulating Theorems II.13 and III.7 of [D] (compare with Theorems VI.13 and VI.24 of [Q] and Theorems 7.3.1 and 7.3.6 of [PF]) we obtain:

Lemma A.1. Let $\sigma$ be a substitution of constant length $q$ and height h. Let $u$ be the sequence generated by $\sigma$. Then $u$ is a regular model set iff $\sigma$ admits a Dekking coincidence. The internal space $H$ is given by $H=\mathbb{Z}_{q} \times C_{h}$, where $\mathbb{Z}_{q}$ denotes the product over the distinct primes $p$ dividing $q$ of the $p$-adic integers $\mathbb{Z}_{p}$ and $C_{h}$ is the cyclic group of order $h$.

We compare this result with the ones involving the modular coincidence (Definition 3.2 and Theorem 3.3): The substitution has a modular coincidence iff it is a regular model set with internal space $C_{r} \times \lim _{\leftarrow k} \mathbb{Z} / q^{k} \mathbb{Z}$. Obviously, $\lim _{\leftarrow k} \mathbb{Z} / q^{k} \mathbb{Z} \simeq \mathbb{Z}_{q}$ (e.g., observe that $\lim _{\leftarrow k} \mathbb{Z} / 4^{k} \mathbb{Z}$ is topologically isomorphic to $\mathbb{Z}_{2}$, wherefore it is enough to consider just the distinct primes $p$ ). We can write $r=h \cdot \tilde{q}$, where $\operatorname{gcd}\{r, q\}=\tilde{q}$, and we observe that $C_{r} \times \mathbb{Z}_{q}$ is topologically isomorphic to $C_{h} \times \mathbb{Z}_{q}$ (note that $C_{\tilde{q}} \times \mathbb{Z}_{q}$ and $\mathbb{Z}_{q}$ are topologically isomorphic). So both methods actually give — up to isomorphism - the same internal space.

In a sense, the height $h$ tells us whether $L^{\prime}=r \cdot \mathbb{Z}$ lines up with the length $q$ of the substitution. If $h=1$ but $r>1$, then we do not have to know the sets $\Phi_{0}[\cdot]$ explicitly for the Dekking coincidence, the substitution rule itself already gives the right partition of the alphabet.

Example (continued). The underlying substitution of the paperfolding sequence ( $a \mapsto$ $a b, b \mapsto c b, c \mapsto a d, d \mapsto c d$ ) has height $h=1$ but $r=2$. We observe that there is either an $a$ or a $c$ on the zeroth place of the substitute, while on the first we have either $b$ or 
$d$. However, we also have $\Psi_{0}[0]=\{a, c\}$ and $\Psi_{0}[1]=\{b, d\}$, therefore the substitution lines up with this partition. We note that this substitution has a modular coincidence relative to $2^{2} \mathbb{Z}$ (after two substitutions the first letter is always an $a, a \mapsto a b \mapsto a b c b$, $b \mapsto c b \mapsto a d c b$ etc.), and therefore is pure point diffractive.

\section{Appendix B. Subdivision Graph for Overlaps}

Instead of the coincidence graph $G_{(\mathbf{V}, \Phi)}$, we can also define a pair coincidence graph $G_{(\mathbf{V}, \Phi)}^{(2)}: G_{(\mathbf{V}, \Phi)}^{(2)}$ is a directed (multi-)graph, where each set of pairs of elements of $\Psi_{0}[a]$ for some $a$ (i.e., both elements are from $\Psi_{0}[a]$ with the same $a$ ) is represented by a vertex. We call pairs of the same element $\{i, i\}=\{i\}$ a coincidence in the pair coincidence graph. We draw an edge from $v_{1}$ to $v_{2}$ iff $v_{2}$ is a "child" of $v_{1}$. (Here we mean by an "child": If $v_{1}=\left\{s_{1}, \ldots, s_{\ell}\right\}$ then $v_{2}=\left\{\Phi\left(s_{1}\right)_{z}, \ldots, \Phi\left(s_{\ell}\right)_{z}\right\}$ for some $z$.) The pair coincidence graph of the table tiling is shown in Fig. 9.

Obviously, if the coincidence graph $G_{(\mathbf{V}, \Phi)}$ has a singleton set (i.e., we have a modular coincidence), then from each vertex of $G_{(\mathbf{V}, \Phi)}^{(2)}$ there is a path leading to a coincidence. Conversely, since the alphabet is finite, if from each vertex of $G_{(\mathbf{V}, \Phi)}^{(2)}$ there is a path leading to a coincidence, then $G_{(\mathbf{V}, \Phi)}$ also has a singleton set (compare this with Corollary 4.7). Therefore, we can also use the pair coincidence graph to decide the question about modular coincidences (the pair coincidence graph of the table tiling is not connected, the coincidences forming one connected component; the table tiling has no modular coincidences).

On the tiling level, the pair coincidence graph is called the subdivision graph for overlaps, see p. 721 of [So] and Appendix 2 of [LMS2]. The construction of this sub-
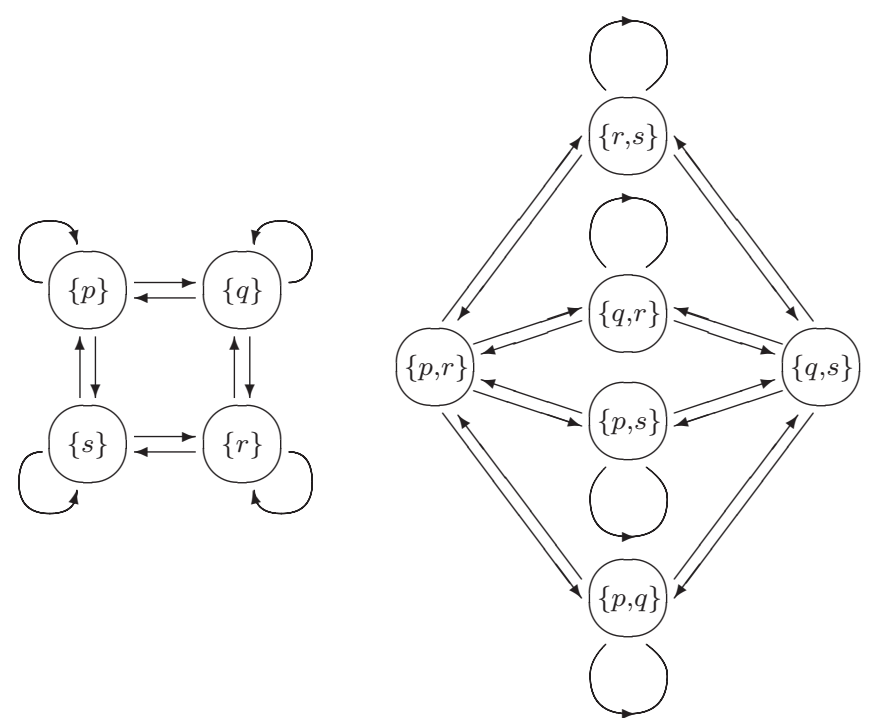

Fig. 9. Pair coincidence graph for the table tiling. 

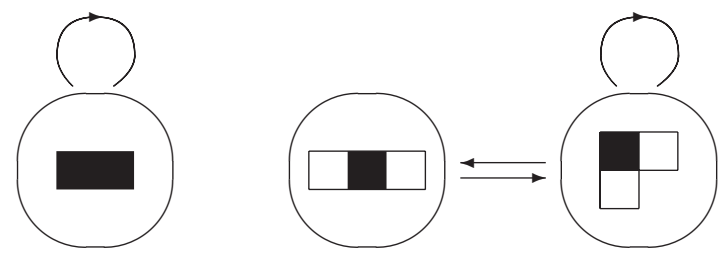

Fig. 10. Subdivision graph for overlaps for the table tiling.

division graph follows closely, with the necessary changes, the construction of the pair coincidence graph. For the pair coincidence graph we can think of placing two multicomponent Delone sets on top of each other (or placing a translated (by a vector of $L^{\prime}$ ) copy of the multi-component set on top) and observing what happens under substitution. Likewise, we can think of placing two tilings on top of each other and then we observe what happens to the overlaps of the tiles under substitution. As an illustration we give the subdivision graph for overlaps (overlaps are denoted by filled areas) for the table tiling in Fig. 10 (where we identify rotated versions of the configurations), see also Fig. 7.4(d) of [So]. This graph should be compared with the pair coincidence graph in Fig. 9 (basically, rotation identifies all the singletons, $\{p, r\}$ and $\{q, s\}$, and the four sets $\{r, s\},\{q, r\},\{p, s\}$ and $\{p, q\})$.

\section{Appendix C. Connections to Automatic Sequences}

In $[\mathrm{H}]$ von Haeseler is interested in the question, under which conditions a sequence is automatic and/or generated by a (not necessarily primitive) substitution of constant length (and in the higher-dimensional case even whether there exists an LSS). To this end, he introduces the kernel of a sequence $u=u_{0} u_{1} u_{2} \ldots$ [H, Definition 2.2.18], which (in one dimension) one can think of as the set $\left\{u_{a} u_{q^{k}+a} u_{2 q^{k}+a} \ldots \mid k \in \mathbb{N}_{0}, a \in\left\{0, \ldots, q^{k}-\right.\right.$ $1\}\}$, i.e., the set of subsequence on $q^{k} \mathbb{Z}+a\left(Q^{k} L+a\right)$ for all $k$ and $a$. The finiteness (and cardinality) of this set is in question, and can be answered (see Lemma 2.2.22 of $[\mathrm{H}])$ by the substitution graph [H, Definition 2.2.20]: The substitution graph is the directed, labeled graph with base point $(1, \ldots, m)$ (the whole alphabet) and the vertices are given by all ordered $m$-tuples $\left(\Phi^{k}(1)_{z^{\prime}}, \ldots, \Phi^{k}(m)_{z^{\prime}}\right)$ (where $z^{\prime} \in L / Q^{k} L$ ), which can be obtained by some power $k$ of the substitution. We draw an edge with label $z$ from $v_{1}=\left(s_{1}, \ldots, s_{m}\right)$ to $v_{2}=\left(\Phi\left(s_{1}\right)_{z}, \ldots, \Phi\left(s_{m}\right)_{z}\right)(z \in L / Q L)$.

Obviously, in the case $L=L^{\prime}$, the coincidence graph can be obtained from the substitution graph: $m$-tuples which are equal as sets are identified. Lemma 4.3 tells us that with this identification there is no problem with the edges. Furthermore, an $m$-tuple $(i, \ldots, i)$ indicates a coincidence. As an example, we give the substitution (see Example 1 following Definition 2.2.20 of [H]) and coincidence graphs of the Thue-Morse sequence $(a \mapsto a b, b \mapsto b a)$ (Fig. 11).

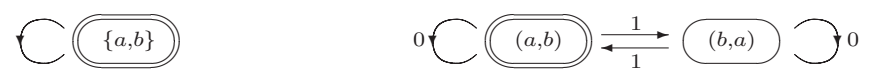

Fig. 11. Coincidence graph (left) and substitution graph (right) of the Thue-Morse substitution. 


\section{Acknowledgements}

Both authors thank the referees for many valuable suggestions. D.F. thanks L. Danzer for helpful discussions about nonadmissible substitutions.

\section{References}

[BL] M. Baake and D. Lenz: Dynamical systems on translation bounded measures: Pure point dynamical and diffraction spectra, Ergodic Theory Dynam. Systems 24 (2004), 1867-1893; math. DS/ 0302061.

[BMS] M. Baake, R.V. Moody and M. Schlottmann: Limit-(quasi)periodic point sets as quasicrystals with p-adic internal spaces, J. Phys. A: Math. Gen. 31 (1998), 5755-5765; math-ph/9901008.

[BSJ] M. Baake, M. Schlottmann and P.D. Jarvis: Quasiperiodic tilings with tenfold symmetry and equivalence with respect to local derivability, J. Phys. A: Math. Gen. 24 (1991), 4637-4654.

[D] F.M. Dekking: The spectrum of dynamical systems arising from substitutions of constant length, Z. Wahrsch. Verw. Gebiete 41 (1978), 221-239.

[F] D. Frettlöh: Nichtperiodische Pflasterungen mit ganzzahligem Inflationsfaktor, Ph.D. Thesis, Dortmund (2002); http: / / hal . handle. net/2003/2309.

[Fra1] N.P. Frank: Substitution sequences in $\mathbb{Z}^{d}$ with a non-simple Lebesgue component in the spectrum, Ergodic Theory Dynam. Systems 23 (2003), 519-532.

[Fra2] N.P. Frank: Multidimensional constant-length substitution sequences, Topology Appl. 152 (2005), 44-69.

[G] J.-B. Gouéré: Quasicrystals and almost periodicity, Comm. Math. Phys. 255 (2005), 655-681; math-ph/0212012.

[H] F. von Haeseler: Automatic Sequences, de Gruyter Expositions in Mathematics, vol. 36, de Gruyter, Berlin, 2003.

[K] S. Kakutani: Strictly ergodic symbolic dynamical systems, Proc. Sixth Berkeley Sympos. Math. Statist. Probab., Univ. Calif. 1970, vol. 2 (1972), pp. 319-326.

[KeP] J. Kellendonk and I.F. Putnam: Tilings, $C^{*}$-algebras and $K$-theory, in: Directions in Mathematical Quasicrystals, M. Baake and R.V. Moody (eds.), CRM Monograph Series, vol. 13, AMS, Providence, RI (2000), pp. 177-206.

[LM] J.-Y. Lee and R.V. Moody: Lattice substitution systems and model sets, Discrete Comput. Geom. 25 (2001), 173-201; math.MG/0002019.

[LMS1] J.-Y. Lee, R.V. Moody and B. Solomyak: Pure point dynamical and diffraction spectra, Ann. Henri Poincaré 3 (2002), 1003-1018; mp_arc / 02 - 39.

[LMS2] J.-Y. Lee, R.V. Moody and B. Solomyak: Consequences of pure point diffraction spectra for multiset substitution systems, Discrete Comput. Geom. 29 (2003), 525-560.

[Ma] K. Mahler: The spectrum of an array and its application to the study of the translation properties of a simple class of arithmetical functions. Part two. On the translation properties of a simple class of arithmetical functions, J. Math. and Phys. (Massachusetts) 6 (1926), 158-163.

[Mo] R.V. Moody: Model sets: a survey, in: From Quasicrystals to More Complex Systems, F. Axel, F. Dénoyer and J.P. Gazeau (eds.), EDP Sciences, Les Ulis, and Springer, Berlin (2000), pp. 145 166; math.MG/ 0002020.

[ORS] N. Ormes, C. Radin and L. Sadun: A homeomorphism invariant for substitution tiling spaces, Geom. Dedicata 90 (2002), 153-182.

[PF] N. Pytheas Fogg: Substitutions in Dynamics, Arithmetics and Combinatorics, Lecture Notes in Mathematics, vol. 1794, Springer, Berlin (2002).

[R] E.A. Robinson, Jr.: On the table and the chair, Indag. Math. (N.S.) 10 (1999), 581-599.

[Q] M. Queffélec: Substitution Dynamical Systems-Spectral Analysis, Lecture Notes in Mathematics, vol. 1294, Springer, Berlin (1987). 
[Sc] M. Schlottmann: Generalized model sets and dynamical systems, in: Directions in Mathematical Quasicrystals, M. Baake and R.V. Moody (eds.), CRM Monograph Series, vol. 13, AMS, Providence, RI (2000), pp. 143-159.

[Si] B. Sing: Kolakoski-(2m, 2n) are limit-periodic model sets, J. Math. Phys. 44 (2003), 899-912; math-ph/0207037.

[So] B. Solomyak: Dynamics of self-similar tilings, Ergodic Theory Dynam. Systems 17 (1997), 695-738. B. Solomyak: Corrections to "Dynamics of self-similar tilings", Ergodic Theory Dynam. Systems 19 (1999), 1685.

Received September 12, 2005, and in revised form March 23, 2006, and May 5, 2006.

Online publication February 28, 2007. 